\title{
An exhaustive study of particular cases leading to robust and accurate motion estimation.
}

\author{
Diane Lingrand \\ INRIA - RobotVis project \\ B.P.9306902 Sophia Antipolis Cédex \\ FRANCE \\ E-mail: Diane.Lingrand@sophia.inria.fr
}

D R A F T April 23, 2001, 5:10pm D R A F T 
For decades, there has been an intensive research effort in the Computer Vision community to deal with video sequences. In this paper, we present a new method for recovering a maximum of information on displacement and projection parameters in monocular video sequences without calibration. This work follows previous studies on particular cases of displacement, scene geometry and camera analysis and focuses on the particular forms of homographic matrices.

It is already known that the number of particular cases involved in a complete study precludes an exhaustive test. To lower the algorithmic complexity, some authors propose to decompose all possible cases in a hierarchical tree data structure but these works are still in development ??. In this paper, we propose a new way to deal with the huge number of particular cases : (i) we use simple rules in order to eliminate some redundant cases and some physically impossible cases, and (ii) we divide the cases into subsets corresponding to particular forms determined by simple rules leading to a computationally efficient discrimination method.

Finally, some experiments were performed on image sequences acquired either using a robotic system or manually in order to demonstrate that when several models are valid, the model with the fewer parameters gives the best estimation, regarding the free parameters of the problem. The experiments presented in this paper shows that even if the selected case is an approximation of reality, the method is still robust.

Key Words: particular cases; homographies; perspective, para-perspective and orthographic projections

\section{INTRODUCTION}

\section{R A F T April 23, 2001, 5:10pm D R A F T}


For decades, there has been an intensive research effort in the Computer Vision community to deal with video sequences. Researchers have been interested in recovering 3D objects structure, projection or displacement parameters from such sequences. In the general case, the acquisition device has to be considered uncalibrated (for example, in the case of an auto-focus camera). In this paper, we consider uncalibrated monocular video sequences for which we intend to recover as much information as possible on displacement and projection parameters.

The motivations for such studies are threefold: (i) to eliminate singularities of general equations, (ii) to estimate the parameters with more robustness and (iii) to retrieve parameters that cannot be retrieved in the general case.

The theory states that there exists relations between 2D projected points [9] but the system cannot be solved in the general case since there are more parameters than equations. Furthermore, these equations are degenerate or present singularities in some particular cases. However, we can solve the equations if we know or assume values or relations of some parameters.

In a previous study [25], we have shown that we increase the numerical precision of retrieved parameters by using the set of constraints that gives the smallest residual error given by a criterion (described in the cited paper).

This paper extends previous works $[25,13,14]$ on particular displacement cases, scene geometry and camera analysis. It focuses on the particular forms of fundamental and homographic matrices.

Several authors have already been interested in particular cases of projection $[2,6,11,22,18]$, or displacement $[10,5,24,3,23]$. Some of them consider several particular cases, compare these different parameterizations and identify which model is consistent with the data.

We will build an exhaustive list of particular cases of projection and displacement, setting some of the parameters to constant and/or known values and using

\section{R A F T April 23, 2001, 5:10pm D R A F T}


known relations between parameters. This reduces the number of unknowns in the equations and avoid also some singular cases.

It is already known that the huge number of particular cases prevents exhaustive studies [13]. Some attempts in order to reduce the algorithmic complexity are based on tree structures but they are still in development [25]. In this paper, we introduce a new method in order to deal with all cases : (i) we use simple rules in order to eliminate some redundant cases and some physically impossible cases, and (ii) we divide the cases into subsets corresponding to particular forms determined by simple rules leading to computationally efficient discrimination method. We will provide details for each of these steps in the sections hereafter.

\section{STEREO FRAMEWORK}

In this section, we describe the equations and the formalism of displacement and projection which allows us to achieve a minimal parameterization of the relations between $2 \mathrm{D}$ points into two frames.

In a video sequence, we will consider frames pairwise: two consecutives frames or the first one and the last one. This work could be easily extended to trifocal tensors. Adding some other constraints, the framework could also be extended to sequences, assuming for examples that the translation is constant between consecutives frames, or varies with constant acceleration, ...

\subsection{Rigid displacements}

We will consider a rigid scene or piecewise rigid scene. A 3D-point $\mathbf{M}_{\mathbf{1}}=$ $\left[\begin{array}{llll}X_{1} & Y_{1} & Z_{1} & 1\end{array}\right]^{T}$ is moving onto the point $\mathbf{M}_{2}=\left[\begin{array}{llll}X_{2} & Y_{2} & Z_{2} & 1\end{array}\right]^{T}$ by a rotation $\mathbf{R}$ and a translation $\mathbf{t}=\left[t_{0} t_{1} t_{2}\right]^{T}: \mathbf{M}_{\mathbf{2}}=\mathbf{R} \mathbf{M}_{\mathbf{1}}+\mathbf{t}$ as shown in figure 1.

A rotation matrix $\mathbf{R}$ depends only on three parameters $\mathbf{r}=\left[\begin{array}{lll}r_{0} & r_{1} & r_{2}\end{array}\right]^{T}$ related to the rotation angle $\theta$ and to the rotation axis direction (represented by the unary vector $\mathbf{u})$ by $\mathbf{r}=2 \tan \left(\frac{\theta}{2}\right) \mathbf{u} \Leftrightarrow \theta=2 \arctan \left(\frac{\|\mathbf{r}\|}{2}\right)$
D R A F T
April 23, 2001, 5:10pm
D R A F T 


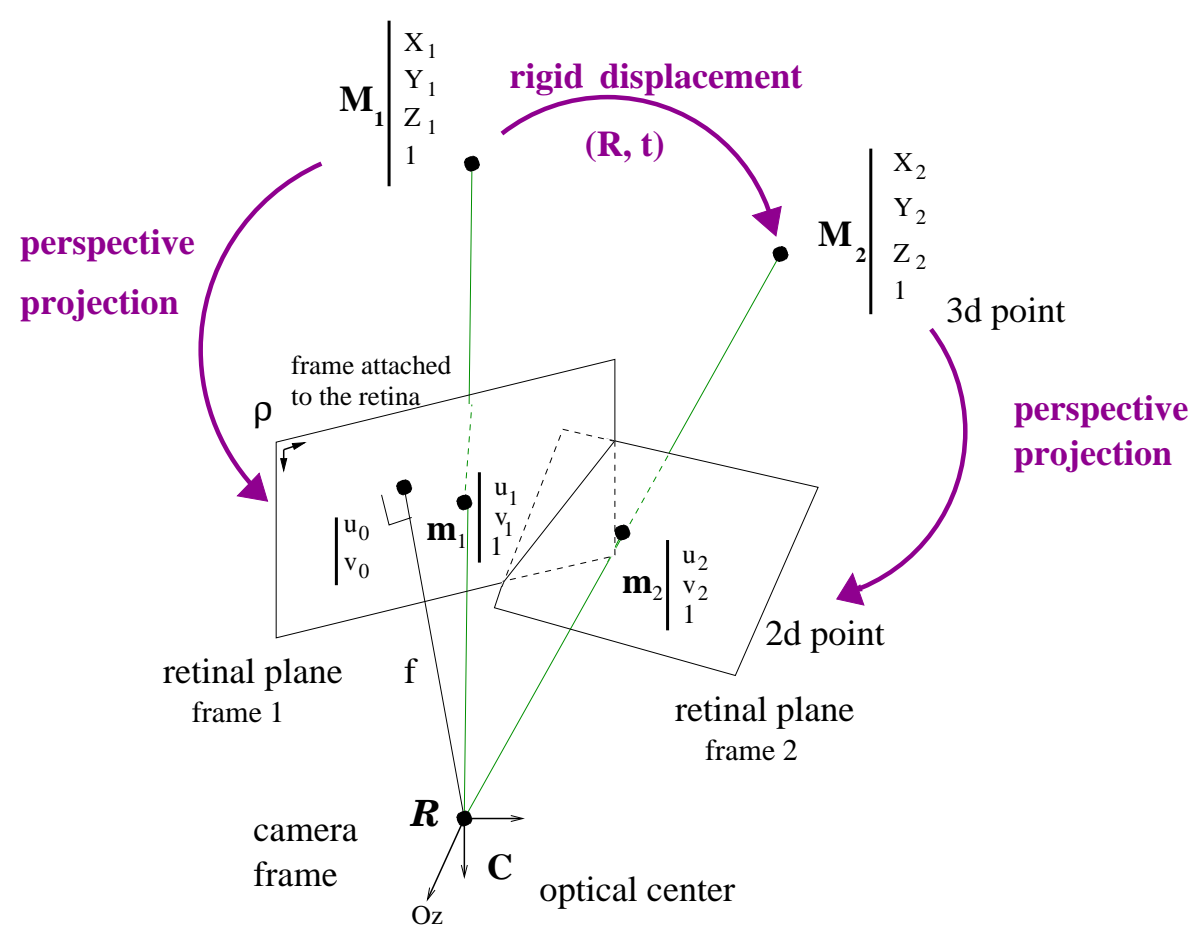

FIG. 1. Stereo framework

Using the notation of the cross-product :

$$
\tilde{\mathbf{r}}=\mathbf{r} \wedge .=\left(\begin{array}{ccc}
0 & -r_{2} & r_{1} \\
r_{2} & 0 & -r_{0} \\
-r_{1} & r_{0} & 0
\end{array}\right)
$$

so that :

$$
\forall \mathrm{x}, \mathrm{r} \wedge \mathrm{x}=\tilde{\mathbf{r}} \mathbf{x}
$$

$\tilde{\mathbf{r}}$ is the antisymmetric matrix representing the cross-product by $\mathbf{r}$ operator.

The rotation matrix $\mathbf{R}=e^{\tilde{\mathbf{r}}}$ can be developed as a rational Rodrigues formula, [19] :

$$
\mathbf{R}=\mathbf{I}+\left[\frac{\tilde{\mathbf{r}}+\frac{1}{2} \tilde{\mathbf{r}}^{2}}{1+\frac{\mathbf{r}^{T} \cdot \mathbf{r}}{4}}\right]
$$

\subsection{Camera projection}

D R A F T April 23, 2001, 5:10pm D R A F T 
The most commonly accepted hypothesis states that a 3D-point $\mathbf{M}$ is projected with a perspective projection onto an image plane on a $2 \mathrm{D}$-point $\mathbf{m}=\left[\begin{array}{lll}u & v & 1\end{array}\right]^{T}$.

The perspective model : Choosing a reference frame attached to the camera, the projection equation is :

$$
Z\left(\begin{array}{l}
u \\
v \\
1
\end{array}\right)=\left(\begin{array}{cccc}
\alpha_{u} & \gamma & u_{0} & 0 \\
0 & \alpha_{v} & v_{0} & 0 \\
0 & 0 & 1 & 0
\end{array}\right)\left(\begin{array}{l}
X \\
Y \\
Z \\
1
\end{array}\right)
$$

where $\alpha_{u}$ and $\alpha_{v}$ represent the horizontal and vertical lengths, $u_{0}$ and $v_{0}$ correspond to the image of the optical center and $\gamma$ is the skew factor.

This model can be refined, by taking optical distortions into account $[21,4,7]$. In this paper, we will consider that the needed corrections have been done as a preprocessing.

Two approximation models of the projection equation 2 have been proposed in the literature : the para-perspective and the ortho-perspective projection.

The para-perspective model : The perspective projection model is approximated to its first order with respect to the $3 \mathrm{D}$ coordinates $[2,17,11]$. This is equivalent to approximating the perspective projection in two steps (see figure 2) : (i) a projection parallel to the gaze direction onto an auxiliary plane $\mathbf{P}_{a}$ which is parallel to the image plane and passes through the scene center $\mathbf{M}_{\mathbf{0}}=\left[\begin{array}{lll}X_{0} & Y_{0} & Z_{0}\end{array}\right]^{T}$ followed by (ii) a perspective projection onto the image plane. This so called para-perspective model yields linear equations (3). However, its parameters depend on the gaze direction of the scene $\left(\beta_{u}\right.$ and $\beta_{v}$ are related to the other intrinsic parameters and to the gaze direction) :
D R A F T
April 23, 2001, 5:10pm
D R A F T 


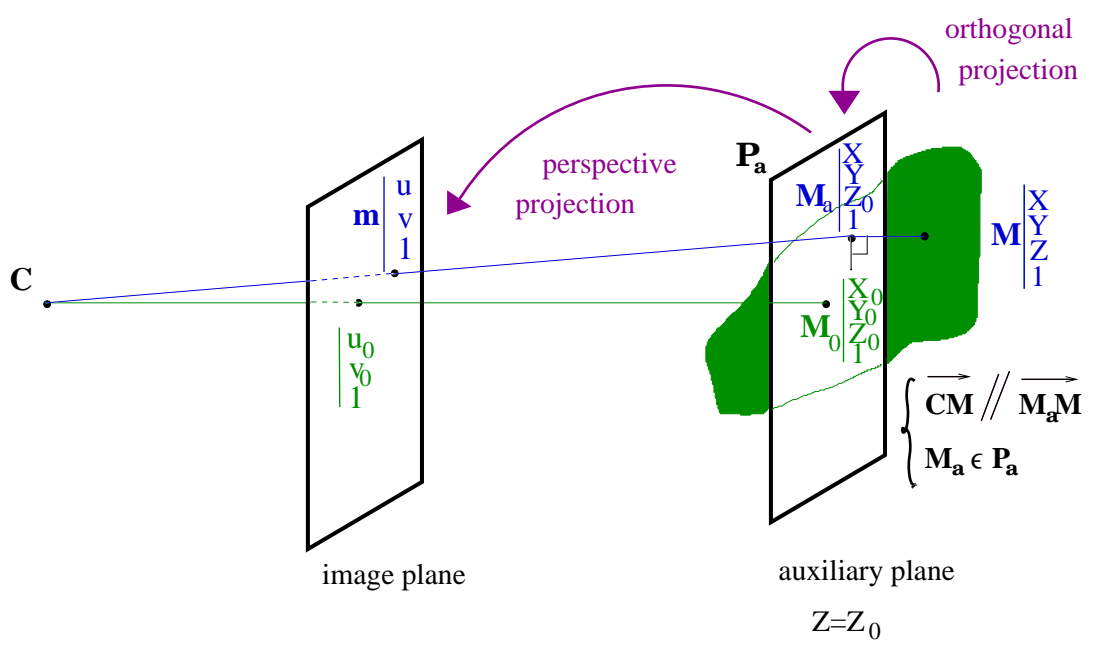

FIG. 2. The para-perspective projection

$$
\left(\begin{array}{l}
u \\
v \\
1
\end{array}\right)=\left(\begin{array}{cccc}
\alpha_{u} & \gamma & \beta_{u} & u_{0} \\
0 & \alpha_{v} & \beta_{v} & v_{0} \\
0 & 0 & 0 & 1
\end{array}\right)\left(\begin{array}{l}
X \\
Y \\
Z \\
1
\end{array}\right)
$$

where :

$$
\left\{\begin{array}{l}
\beta_{u}=\alpha_{u} \frac{X_{0}}{Y_{0}}+\gamma \frac{Y_{0}}{Z_{0}} \\
\beta_{v}=\alpha_{v} \frac{Y_{0}}{Z_{0}}
\end{array}\right.
$$

This equation corresponds to the most general case of para-perspective projection although more simple expressions have been proposed [18].

The orthographic model : The zero-order development with respect to the $3 \mathrm{D}$ depth consists in a rougher approximation. It is also equivalent to another two-step approximation: (i) an orthogonal projection onto the auxiliary plane $\mathbf{P}_{a}$ followed by (ii) a perspective projection onto the image plane (see figure 3 ). This approxi-
D R A F T
April 23, 2001, 5:10pm
D R A F T 


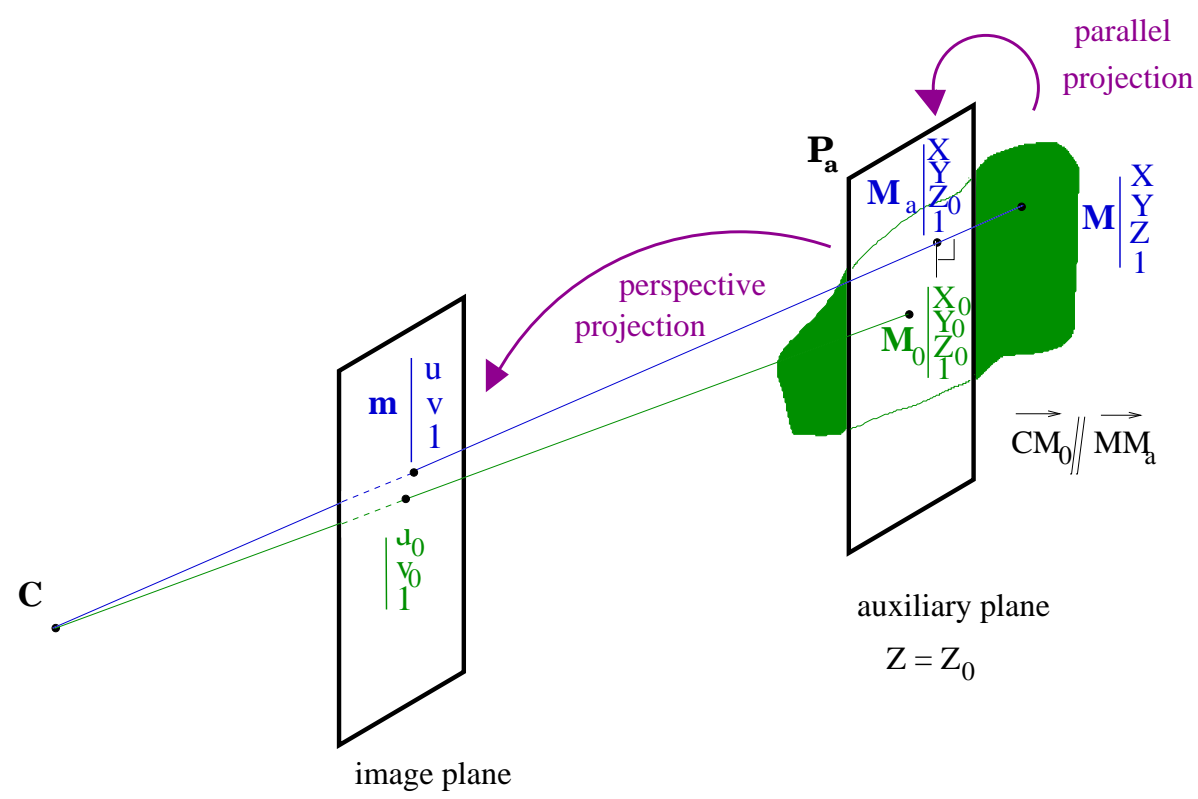

FIG. 3. The orthographic projection

mation, called the orthographic model (4), is well adapted to foveal attention and is characterized by linear equations without any new parameters. It is an approximation of the para-perspective model when the observed objects are in the fovea, i.e. close to the optical axis :

$$
\left(\begin{array}{l}
u \\
v \\
1
\end{array}\right)=\left(\begin{array}{cccc}
\alpha_{u} & \gamma & 0 & u_{0} \\
0 & \alpha_{v} & 0 & v_{0} \\
0 & 0 & 0 & 1
\end{array}\right)\left(\begin{array}{l}
X \\
Y \\
Z \\
1
\end{array}\right)
$$

Those three projection models can be integrated in the following expression :

$$
\kappa \mathbf{m}=\underbrace{\left(\begin{array}{cccc}
\alpha_{u} & \gamma & \lambda \beta_{u}+\mu u_{0} & (1-\mu) u_{0} \\
0 & \alpha_{v} & \lambda \beta_{v}+\mu v_{0} & (1-\mu) v_{0} \\
0 & 0 & \mu & (1-\mu)
\end{array}\right)}_{\mathbf{A}} \mathbf{M}
$$

with :

D R A F T April 23, 2001, 5:10pm D R A F T 


\begin{tabular}{|l|l|l|}
\hline projection case & $\boldsymbol{\lambda}$ & $\boldsymbol{\mu}$ \\
\hline perspective projection & 1 & 1 \\
orthographic projection & 0 & 0 \\
para-perspective projection & 1 & 0 \\
\hline
\end{tabular}

\subsection{General equations between two frames}

Let $I_{1}$ and $I_{2}$ denote two images. In the general case, there exists a fundamental relation between a point $\mathbf{m}_{\mathbf{2}}$ in $I_{2}$ and its corresponding $\mathbf{m}_{\mathbf{1}}$ in $I_{1}$ :

$$
\left\{\begin{array}{c}
\kappa_{1} \mathbf{m}_{\mathbf{1}}=\mathbf{A}_{\mathbf{1}} \mathbf{M}_{\mathbf{1}} \\
\kappa_{2} \mathbf{m}_{\mathbf{2}}=\mathbf{A}_{\mathbf{2}} \mathbf{M}_{\mathbf{2}} \\
\mathbf{M}_{\mathbf{2}}=\mathbf{R} \mathbf{M}_{\mathbf{1}}+\mathbf{t}
\end{array}\left|\left(\begin{array}{l}
\mathbf{m}_{\mathbf{1}} \\
0 \\
0 \\
0
\end{array}\right) \begin{array}{ll}
0 \\
\mathbf{m}_{2} & \mathbf{A}_{\mathbf{2}}[\mathbf{R} \| \mathbf{t}] \\
\end{array}\right|=0\right.
$$

which is a bilinear form in $\mathbf{m}_{\mathbf{1}}$ and $\mathbf{m}_{\mathbf{2}}$ (see [12] for details). This equation can be rewritten in a more common way :

$$
\mathbf{m}_{\mathbf{2}}^{T} \mathbf{F} \mathbf{m}_{\mathbf{1}}=\mathbf{0}
$$

where $\mathbf{F}$ is called the fundamental matrix [9].

However, this relation is not defined in some singular cases. For example, it is well known that, in the perspective projection case, if the displacement is a pure rotation, or if the scene is planar, the relation between points is homographic :

$$
\mathbf{m}_{2}=\mathbf{H} \mathbf{m}_{1}
$$

where $\mathbf{H}$ is called the homographic matrix. In the case of a pure rotation : $\mathbf{H}=$ $\mathbf{H}_{\infty}=\mathbf{A}_{\mathbf{2}} \mathbf{R} \mathbf{A}_{\mathbf{1}}^{-\mathbf{1}}$. In the case of a plane with normal $\mathbf{n}$ and distance to the origin $d: \mathbf{H}=\mathbf{A}_{\mathbf{2}}\left(\mathbf{R}+\frac{\mathbf{t n}^{\mathrm{T}}}{d}\right) \mathbf{A}_{\mathbf{1}}{ }^{-1}$ which goes to $\mathbf{H}_{\infty}$ when $d$ goes to $\infty . \mathbf{H}_{\infty}$ is the homography of the plane at infinity.
D R A F T
April 23, 2001, 5:10pm
D R A F T 
Our first new contribution in this paper will be explained in the two next paragraphs 2.4 and 2.5. It consists in determining in which case of displacement or structure, the relation between corresponding $2 \mathrm{D}$ points is homographic when the projection is para-perspective (2.4) or orthographic (2.5).

\subsection{Homographic relation in the para-perspective case}

In the para-perspective case, we write the projection and displacement equations by extracting the third column from matrix $\mathbf{A}$ :

$$
\left(\begin{array}{l}
u \\
v \\
1
\end{array}\right)=\underbrace{\left(\begin{array}{ccc}
\alpha_{u} & \gamma & u_{0} \\
0 & \alpha_{v} & v_{0} \\
0 & 0 & 1
\end{array}\right)}_{(\mathbf{A})_{-3}} \underbrace{\left(\begin{array}{l}
X \\
Y \\
1
\end{array}\right)}_{\underline{\mathbf{M}}}+Z \underbrace{\left(\begin{array}{c}
\beta_{u} \\
\beta_{v} \\
1
\end{array}\right)}_{(\mathbf{A})_{3}}=(\mathbf{A})_{-3} \underline{\mathbf{M}}+Z(\mathbf{A})_{3}
$$

where $(\mathbf{A})_{-3}$ is an invertible square matrix since :

$$
\operatorname{det}\left((\mathbf{A})_{-3}\right)=\alpha_{u} \alpha_{v} \neq 0
$$

Thus :

$$
\left\{\begin{array}{l}
\mathbf{m}_{\mathbf{1}}=\left(\mathbf{A}_{\mathbf{1}}\right)_{-3} \underline{\mathbf{M}}_{\mathbf{1}}+Z_{1}\left(\mathbf{A}_{\mathbf{1}}\right)_{3} \\
\Rightarrow \underline{\mathbf{M}}_{\mathbf{1}}=\left(\left(\mathbf{A}_{\mathbf{1}}\right)_{-3}\right)^{-1} \mathbf{m}_{\mathbf{1}}-Z_{1}\left(\left(\mathbf{A}_{\mathbf{1}}\right)_{-3}\right)^{-1}\left(\mathbf{A}_{\mathbf{1}}\right)_{3} \\
\mathbf{m}_{\mathbf{2}}=\mathbf{A}_{\mathbf{2}} \mathbf{M}_{\mathbf{2}} \\
\mathbf{M}_{\mathbf{2}}=[\mathbf{R} \mid \mathbf{t}] \mathbf{M}_{\mathbf{1}}
\end{array}\right.
$$

Let us denote :

$$
\begin{aligned}
\mathbf{K} & =\left(\mathbf{A}_{\mathbf{2}}[\mathbf{R} \mid \mathbf{t}]\right)_{3}-\left(\mathbf{A}_{\mathbf{2}}[\mathbf{R} \mid \mathbf{t}]\right)_{-3}\left(\left(\mathbf{A}_{\mathbf{1}}\right)_{-3}\right)^{-1}\left(\mathbf{A}_{\mathbf{1}}\right)_{3} \\
\text { and } \quad \mathbf{H}_{\infty_{\text {para }}} & =\left(\mathbf{A}_{\mathbf{2}}[\mathbf{R} \mid \mathbf{t}]\right)_{-3}\left(\left(\mathbf{A}_{\mathbf{1}}\right)_{-3}\right)^{-1}
\end{aligned}
$$

Equation 6 leads to : $\mathbf{m}_{\mathbf{2}}=\mathbf{H}_{\infty_{\text {para }}} \mathbf{m}_{\mathbf{1}}+Z_{1} \mathbf{K}$

This relation is homographic if and only if $\mathbf{K}=0$ or if there exists a $(3 \times 3)$ matrix $\mathbf{H}_{\mathbf{Z}}$ such that $Z_{1} \mathbf{K}=\mathbf{H}_{\mathbf{Z}} \mathbf{m}_{\mathbf{1}}$. The first condition induces a displacement constraint. It leads to the simple equation $\mathbf{r}=\theta \mathbf{M}_{\mathbf{0}}$ meaning that the rotation axis is parallel to the gaze direction. In that case, the homography is $\mathbf{H}_{\infty_{\text {para }}}$ as
D R A F T
April 23, 2001, 5:10pm
D R A F T 
defined above. The second condition induces a geometric relation on the 3D point :

$Z_{1}$ is an affine function of $X_{1}$ and $Y_{1}$, meaning that the $3 \mathrm{D}$ points must belong to a plane $\mathbf{P}$, which cannot contain the optical axis and the gaze direction (see [12] for a demonstration). In that case, the homographic matrix is :

$$
\begin{aligned}
\mathbf{H}_{\text {para }} & =\mathbf{H}_{\infty_{\text {para }}} \\
& +\left[\left(\mathbf{A}_{\mathbf{2}}[\mathbf{R} \mid \mathbf{t}]\right)_{3}-\left(\mathbf{A}_{\mathbf{2}}[\mathbf{R} \mid \mathbf{t}]\right)_{-3}\left(\left(\mathbf{A}_{\mathbf{1}}\right)_{-3}\right)^{-1}\left(\mathbf{A}_{\mathbf{1}}\right)_{3}\right] \mathbf{n}\left[\left(\mathbf{A}_{\mathbf{1}}\right)_{-3}+\left(\mathbf{A}_{\mathbf{1}}\right)_{3} \mathbf{n}\right]^{-1}
\end{aligned}
$$

\subsection{Homographic relation in the orthographic case}

The orthographic case is a particular case of para-perspective projection for which the gaze direction is the optical axis. Following a demonstration similar to the paraperspective case, we also obtain two constraints; the displacement constraint states that the rotation axis must be parallel to the optical axis, giving a homographic matrix :

$$
\mathbf{H}_{\infty_{\text {ortho }}}=\left(\mathbf{A}_{\mathbf{2}}[\mathbf{R} \mid \mathbf{t}]\right)_{-3}\left(\left(\mathbf{A}_{\mathbf{1}}\right)_{-3}\right)^{-1}
$$

and the geometric constraint states that the 3D-points must belong to the same plane which does not contain the optical axis. The homographic matrix is :

$$
\mathbf{H}_{\text {ortho }}=\mathbf{H}_{\infty_{\text {ortho }}}+\left(\mathbf{A}_{\mathbf{2}}[\mathbf{R} \mid \mathbf{t}]\right)_{3} \mathbf{n}^{T}\left(\left(\mathbf{A}_{\mathbf{1}}\right)_{-3}\right)^{-1}
$$

All constraints on displacement and scene geometry for homographic relations are summarized in the following table :

\begin{tabular}{|l|c|l|}
\hline projection & displacement constraint & geometric constraint \\
\hline perspective & $\mathbf{t}=\mathbf{0}$ & plane \\
\hline para-perspective & $\mathbf{r} \| \mathbf{C M}_{\mathbf{0}}$ & plane $Z=f(X, Y)$ \\
\hline orthographic & $\mathbf{r} \| \mathbf{0 z}$ & plane $Z=f(X, Y)$ \\
\hline
\end{tabular}
D R A F T
April 23, 2001, 5:10pm
D $R$ A A 


\section{ALL PARTICULAR CASES DESCRIPTION}

In order to do an exhaustive study of particular cases combinations, we first study every elementary particular case. We begin with particular camera parameter values, and then particular displacements of the camera.

\subsection{Particular cases of projection and intrinsic parameters}

In the previous section 2.2, we studied particular cases of projection and their simplifications. Let $\mathrm{p} 1, \mathrm{p} 2$ and $\mathrm{p} 3$ denote the different kinds of projection :

\begin{tabular}{|l|l|l|}
\hline p1 & $\lambda=0$ and $\mu=0$ & orthographic \\
p2 & $\lambda=1$ and $\mu=0$ & para-perspective projection \\
p3 & $\lambda=1$ and $\mu=1$ & perspective projection \\
\hline
\end{tabular}

If no auto-focus and no zoom is used, for instance, it is possible to parameterize the model with fewer parameters than in the general case. This is one reason to study particular cases of intrinsic parameters.

Authors generally make several hypotheses regarding intrinsic parameters. For example, usually, in case of auto-calibration, common hypothesis states that the intrinsic parameters are constant. They may or may not be known. But, usually, some parameters are constant and some other are not.

We now detail all prior knowledge on parameters leading to particular cases.

\subsubsection{The principal point}

The principal point of coordinates $\left(u_{0}, v_{0}\right)$ is not fixed at the image plane in the general case but can be fixed in some cases and its position can be known (for example, at the image center). We then change the reference frame, regarding the principal point position.

\subsubsection{The $\gamma$ parameter}

D R A F T April 23, 2001, 5:10pm D R A F T 
This parameter is usually assumed to be null or, at least, considered a constant value. Furthermore, the numerical precision of the model obtained by this parameter is not crucial for the para-perspective or the orthographic projection cases.

\subsubsection{The $\alpha_{u}$ and $\alpha_{v}$ parameters}

Enciso [8] has experimentally proven that, for a large number of cameras, the $\frac{\alpha_{u}}{\alpha_{v}}$ ratio can be considered as a constant value even if other intrinsic parameters change. The constancy of this ratio can be expressed by the equality $f=\alpha_{u}=\alpha_{v}$, and the following transformation :

$$
\left(\begin{array}{cccc}
\alpha_{u} & \gamma & \lambda \beta_{u}+\mu u_{0} & (1-\mu) u_{0} \\
0 & \alpha_{v} & \lambda \beta_{v}+\mu v_{0} & (1-\mu) v_{0} \\
0 & 0 & \mu & (1-\mu)
\end{array}\right)=\left(\begin{array}{cccc}
f & \gamma & \lambda \beta_{u}+\mu u_{0} & (1-\mu) u_{0} \\
0 & f & \lambda \beta_{v}+\mu v_{0} & (1-\mu) v_{0} \\
0 & 0 & \mu & (1-\mu)
\end{array}\right) \cdot\left(\begin{array}{cccc}
\frac{\alpha_{u}}{\alpha_{v}} & 0 & 0 & 0 \\
0 & 1 & 0 & 0 \\
0 & 0 & 1 & 0 \\
0 & 0 & 0 & 1
\end{array}\right)
$$

\subsubsection{The $\beta_{u}$ and $\beta_{v}$ parameters.}

These parameters are null except in the para-perspective projection case.

In the para-perspective case, $\beta_{u}$ and $\beta_{v}$ are related to the other intrinsic parameters by :

$$
\left\{\begin{array}{l}
\beta_{u}=\alpha_{u} \frac{X_{0}}{Z_{0}}+\gamma \frac{Y_{0}}{Z_{0}} \\
\beta_{v}=\alpha_{v} \frac{Y_{0}}{Z_{0}}
\end{array}\right.
$$

Their ratio is : $\frac{\beta_{u}}{\beta_{v}}=\frac{\alpha_{u} X_{0}+\gamma Y_{0}}{\alpha_{v} Y_{0}}$.

Thus, if we neglect $\gamma$ with respect to $\alpha_{u} \frac{X_{0}}{Y_{0}}$, we obtain : $\frac{\beta_{u}}{\beta_{v}}=\frac{\alpha_{u}}{\alpha_{v}} \frac{X_{0}}{Y_{0}}$ which is also a constant ratio, known if $\frac{X_{0}}{Y_{0}}$ value is known.

Table 1 summarizes, for each intrinsic parameter, the particular cases (constant value are indexed by zero).

Subsequently, we refer to each case by the label given in the first column.
D R A F T
April 23, 2001, 5:10pm
D R A F T 
TABLE 1

Particular cases of intrinsic parameters for 2 frames

\begin{tabular}{|c|l|l|}
\hline label & case & description \\
\hline $\mathrm{g} 1$ & $\gamma=0$ & $\gamma$ constant and null \\
$\mathrm{g} 2$ & $\gamma=\gamma_{0}$ & $\gamma$ constant \\
$\mathrm{g} 3$ & $\gamma=\gamma(\tau)$ & $\gamma$ free \\
\hline $\mathrm{f} 1$ & $\alpha_{v}=1$ & $\alpha_{v}$ constant and known \\
$\mathrm{f} 2$ & $\alpha_{v}=f_{0}$ & $\alpha_{v}$ constant \\
$\mathrm{f} 3$ & $\alpha_{v}=\alpha_{v}(\tau)$ & $\alpha_{v}$ free \\
\hline $\mathrm{s} 1$ & $\alpha_{u}=\alpha_{v}(\tau)$ & $\frac{\alpha_{u}}{\alpha_{v}}$ constant and known \\
$\mathrm{s} 2$ & $\alpha_{u}=\alpha_{u}(\tau)$ & $\alpha_{u}$ free \\
\hline $\mathrm{b} 1$ & $\beta_{v}=0$ & $\beta_{v}$ constant and null \\
$\mathrm{b} 2$ & $\beta_{v}=\beta_{0}$ & $\beta_{v}$ constant \\
$\mathrm{B} 32$ & $\beta_{v}=\beta_{v}(\tau)$ & $\beta_{v}$ free \\
\hline $\mathrm{B} 1$ & $\beta_{u}=\beta_{v}(\tau)$ & $\beta_{v}$ and $\beta_{v}$ equal \\
\hline $\mathrm{B} 1$ & $\beta_{u}=\beta_{u}(\tau)$ & $u_{0}$ constant \\
\hline$\beta_{v}$ free
\end{tabular}

\section{R A F T April 23, 2001, 5:10pm D R A F T}




\subsection{Particular cases of displacement}

A rigid displacement is parameterized by the rotation $\mathbf{R}$ and the translation $\mathbf{t}$ parameters.

\subsubsection{Discrete motion - continuous motion}

In an image sequence, if the displacement between two frames is small, we can approximate the rotation equations by their first order expansion, using the notations $\mathbf{r}=\theta \mathbf{u}:$

$$
\mathbf{R}=e^{\tilde{\mathbf{r}}}=\mathbf{I}+\tilde{\mathbf{r}}+o(\tilde{\mathbf{r}})=\left(\begin{array}{ccc}
1 & -r_{2} & r_{1} \\
r_{2} & 1 & -r_{0} \\
-r_{1} & r_{0} & 1
\end{array}\right)
$$

Otherwise, if the motion is larger, we can also consider the second order expansion :

$$
\mathbf{R}=\mathbf{I}+\tilde{\mathbf{r}}+\frac{\tilde{\mathbf{r}}^{2}}{2}+o\left(\tilde{\mathbf{r}}^{2}\right)=\left(\begin{array}{ccc}
1-\left(r_{1}^{2}+r_{2}^{2}\right) & r_{1} r_{0}-r_{2} & r_{2} r_{0}+r_{1} \\
r_{1} r_{0}+r_{2} & 1-\left(r_{0}^{2}+r_{2}^{2}\right) & r_{2} r_{1}-r_{0} \\
r_{2} r_{0}-r_{1} & r_{2} r_{1}+r_{0} & 1-\left(r_{0}^{2}+r_{1}^{2}\right)
\end{array}\right)
$$

\subsubsection{About extrinsic parameters}

The rotation parameters are related to the rotation axis and the rotation angle by : $\mathbf{r}=2 \tan \frac{\theta}{2} \mathbf{u}$, in the general case and $\mathbf{r}=\theta \mathbf{u}$, in the first or second order of expansion. The vector $\mathbf{u}$ is an unary vector giving the direction of the rotation axis.

Some components of $\mathbf{u}$ can be known or assumed null. Some values of $\theta$ may yield singularities; for example $\theta=0$ corresponds to a null rotation. Another particular case is the screw displacement for which $\theta=\frac{\pi}{4}$ and the rotation axis is parallel to the translation vector. The case $\theta=\pi$ is not considered in this paper but must be considered if the camera has an angle of view greater than 180 degrees.

Some robotic systems give precise values of the robot displacements (angle, axis, translation). Some values may be known (we denote by $\_\theta_{0}$ a constant and known

\section{R A F T April 23, 2001, 5:10pm D R A F T}


value of a parameter $\theta$ ). Other informations on parallelism or orthogonality to a known direction may be available. As is the case for the translation vector.

\subsubsection{Relations between axis and direction}

These relations in which we are interested are orthogonality and parallelism :

- the rotation axis is orthogonal to the translation plane (e.g. planar motion) : $\mathbf{r} \perp \mathbf{t} \Leftrightarrow \mathbf{r . t}=0 \Leftrightarrow r_{0} t_{0}+r_{1} t_{1}+r_{2} t_{2}=0$

- screw displacement : $\mathbf{r} \| \mathbf{t} \Leftrightarrow \exists \kappa / \mathbf{r}=\kappa \mathbf{t} \Leftrightarrow \exists \kappa /\left\{\begin{array}{l}r_{0}=\kappa t_{0} \\ r_{1}=\kappa t_{1} \\ r_{2}=\kappa t_{2}\end{array}\right.$

- the rotation axis or the translation direction is parallel or orthogonal to a known direction denoted by _ $\mathbf{g}\left({ }_{-} \mathbf{r}\right.$ or $\left.\_\mathbf{t}\right)$.

\subsubsection{All constraints on motion}

All these constraints, also called "atomic particular cases", have simple expressions that can easily be combined. For this purpose, we use the fact that $\mathbf{u}$ is an unary vector and that, for monocular systems, the norm of translation cannot be recovered. To parameterize these vectors with only 2 parameters, we divide each component by a non-zero component. Then, the dot-product and scalar product induce linear relations. For example, if $t_{2}=1, \mathbf{t} \perp \mathbf{r}$ is equivalent to $t_{0} u_{0}+t_{1} u_{1}+u_{2}=0 \Rightarrow u_{2}=-t_{0} u_{0}-t_{1} u_{1}$

All cases are collected in table 2.

\subsection{Generation of all cases}

In this section, we combine all previous constraints in order to generate all possible cases. We then generate the simplified equations of our vision problem, i.e., the $\mathrm{F}$ or $\mathrm{H}$ matrix coefficients, depending of the cases.

We call atomic case each case described above. By combining atomic cases, we produce molecular cases, i.e. all possible particular cases. For each molecular case,
D R A F T
April 23, 2001, 5:10pm
D R A F T 
TABLE 2

Particular cases of displacements

\begin{tabular}{|c|c|c|c|c|c|}
\hline $\mathrm{R} 1$ & $\mathbf{R}=\mathbf{I}$ & null rotation & W1 & r. $\_r=0$ & axis $\perp$ known axis \\
\hline R2 & $\mathbf{R}=\mathbf{I}+\tilde{\mathbf{r}}$ & first order & W2 & $\mathbf{r} \wedge{ }_{-} \mathbf{r}=0$ & axis $\|$ known axis \\
\hline R3 & $\mathbf{R}=\mathbf{I}+\tilde{\mathbf{r}}+\frac{1}{2} \tilde{\mathbf{r}}^{2}$ & second order & W3 & & general case \\
\hline $\mathrm{R} 4$ & $\mathbf{R}=\mathbf{I}+\frac{\tilde{\mathbf{r}}+\frac{1}{2} \tilde{\mathbf{r}}^{2}}{1+\frac{\mathbf{r}^{T} \mathbf{r}}{4}}$ & general case & u1 & $u_{0}=u_{2}=0, u_{1}=1$ & axis $\| y$-axis \\
\hline r1 & $\mathbf{r}=2 \tan \left(\frac{\theta}{2}\right) \frac{\mathbf{u}}{\|\mathbf{u}\|}$ & general case & u2 & $u_{0}=0, u_{1}=1$ & axis $\perp$ x-axis \\
\hline a1 & $\theta=\frac{\pi}{2}$ & quarter turn & u3 & $u_{2}=0, u_{1}=1$ & axis $\perp$ z-axis \\
\hline $\mathrm{a} 2$ & $\theta$ & free angle & $\mathrm{u} 4$ & $u_{1}=1$ & general case \\
\hline $\mathrm{T} 1$ & $\mathbf{t}=\mathbf{0}$ & null translation & u 5 & $u_{0}=u_{2}=0, u_{1}=-1$ & axis $\| y$-axis \\
\hline $\mathrm{T} 2$ & $\mathbf{t}=\left[\begin{array}{lll}t_{0} & t_{1} & t_{2}\end{array}\right]^{T}$ & translation & u6 & $u_{0}=0, u_{1}=-1$ & axis $\perp \mathrm{x}$-axis \\
\hline t1 & $t_{1}=t_{2}=0, t_{0}=1$ & trans. $\| \mathrm{x}$-axis & u7 & $u_{2}=0, u_{1}=-1$ & axis $\perp$ z-axis \\
\hline $\mathrm{t} 2$ & $t_{1}=0, t_{0}=1$ & trans. $\perp$ y-axis & u8 & $u_{1}=-1$ & general case \\
\hline $\mathrm{t} 3$ & $t_{2}=0, t_{0}=1$ & trans. $\perp$ z-axis & u9 & $u_{0}=u_{1}=0, u_{2}=1$ & axis \| z-axis \\
\hline $\mathrm{t} 4$ & $t_{0}=1$ & general trans. & $\mathrm{u} 10$ & $u_{0}=0, u_{2}=1$ & axis $\perp x$-axis \\
\hline $\mathrm{t} 5$ & $t_{0}=t_{2}=0, t_{1}=1$ & trans. $\| \mathrm{y}$-axis & u11 & $u_{1}=0, u_{2}=1$ & axis $\perp$ y-axis \\
\hline t6 & $t_{0}=0, t_{1}=1$ & trans. $\perp$ x-axis & u12 & $u_{2}=1$ & general case \\
\hline $\mathrm{t} 7$ & $t_{2}=0, t_{1}=1$ & trans. $\perp$ z-axis & u13 & $u_{0}=u_{1}=0, u_{2}=-1$ & axis $\|$ z-axis \\
\hline $\mathrm{t} 8$ & $t_{1}=1$ & general trans. & u14 & $u_{0}=0, u_{2}=-1$ & axis $\perp \mathrm{x}$-axis \\
\hline t9 & $t_{0}=t_{1}=0, t_{2}=1$ & trans. $\| \mathrm{z}$-axis & $\mathrm{u} 15$ & $u_{1}=0, u_{2}=-1$ & axis $\perp$ y-axis \\
\hline t10 & $t_{0}=0, t_{2}=1$ & trans. $\perp$ x-axis & u16 & $u_{2}=-1$ & general case \\
\hline t11 & $t_{1}=0, t_{2}=1$ & trans. $\perp$ y-axis & u17 & $u_{1}=u_{2}=0, u_{0}=1$ & axis $\| x$-axis \\
\hline $\mathrm{t} 12$ & $t_{2}=1$ & general trans. & u18 & $u_{1}=0, u_{0}=1$ & axis $\perp$ y-axis \\
\hline D1 & t._t $\mathbf{t}=0$ & trans. $\perp$ known axis & u19 & $u_{2}=0, u_{0}=1$ & axis $\perp$ z-axis \\
\hline D2 & $\mathbf{t} \wedge \mathbf{t}_{\mathbf{t}}=0$ & trans. $\|$ known axis & $\mathrm{u} 20$ & $u_{0}=1$ & general case \\
\hline D3 & & no relation & u21 & $u_{1}=u_{2}=0, u_{0}=-1$ & axis $\| \mathrm{x}$-axis \\
\hline $\mathrm{Z} 1$ & $\mathbf{t} . \mathbf{u}=0$ & trans. $\perp$ rotat. axis & $\mathrm{u} 22$ & $u_{1}=0, u_{0}=-1$ & axis $\perp$ y-axis \\
\hline $\mathrm{Z} 2$ & $\mathbf{t} \wedge \mathbf{u}=0$ & screw displacement & u23 & $u_{2}=0, u_{0}=-1$ & axis $\perp$ z-axis \\
\hline Z3 & & no relation & $\mathrm{u} 24$ & $u_{0}=-1$ & general case \\
\hline
\end{tabular}
D R A F T
April 23, 2001, 5:10pm
D R A F T 
constraints are solved by combining the atomic cases and solving the constraints by substitution $^{1}$ with some rules : one projection mode, one rotation mode... This corresponds to choosing one case in each family, a family being named by a label. For example, in the $\mathrm{R}$ family, we have to choose one of R1, R2, R3 and R4. We denote by $R[1-3]$ the set $\{R 1, R 2, R 3\}$ and by $R[1 ; 3]$ the set $\{R 1, R 3\}$. Thus, a molecular case is identified by the sequence :

$\mathrm{p}[1-3] \mathrm{g}[1-3] \mathrm{f}[1-3] \mathrm{s}[1-3] \mathrm{b}[1-3] \mathrm{B}[1-3] \mathrm{c}[1-3] \mathrm{R}[1-4] \mathrm{r} 1 \mathrm{a}[1-2] \mathrm{u}[1-24] \mathrm{W}[1-3] \mathrm{T}[1-2] \mathrm{t}[1-12] \mathrm{D}[1-3] \mathrm{Z}[1-3]$

\subsubsection{How many cases do we have?}

If we look at the expression of a particular case above-mentioned, we obtain $3.10^{8}$ particular cases. But, this is not the real number of particular cases due to :

Incompatibility of some atomic cases, for instance (the symbol $\otimes$ means "AND”) :

$$
(\mathbf{r} \| \mathbf{t}) \otimes(\mathbf{r} \perp \mathbf{t}) \otimes(\mathbf{r} \neq 0) \otimes(\mathbf{t} \neq 0)
$$

Redundancy of some constraints; two different set of atomic constraints can generate the same simplified model. For instance :

$$
\left(r_{0}=0\right) \otimes(\mathbf{t} \perp \mathbf{r}) \text { is the same case than }\left(t_{1}=0\right) \otimes\left(t_{2}=0\right) \otimes(\mathbf{t} \perp \mathbf{r})
$$

It is easy to eliminate incompatible constraints. To deal with redundant constraints requires to compare each set of combined constraint with the others in order to determine the similarity. The complexity of this process is $O\left(n^{2}\right)$, that makes this elimination intractable for large values of $n$.

Furthermore, redundant cases are not the main reason for the big amount of particular cases. Thus suppressing redundancies is not sufficient to reduce the huge number of case to a computationally tractable amount.

We now propose an adapted strategy in order to deal with all cases. Previous works have tried to build a hierarchy of cases but they encounter problems in order

\footnotetext{
${ }^{1}$ This work is done using Maple for symbolic computation.
}

\section{R A F T April 23, 2001, 5:10pm D R A F T}


to manage it. The idea of this paper is (i) to eliminate some of the redundant cases by some considerations on the atomic cases and (ii) to limit the number of cases by the study of the particular forms of the matrices. For this second step, we will separate cases into two subgroups: cases inducing homographies and cases inducing fundamental relations.

\subsection{Reducing the number of cases}

Some redundancies are obvious :

- in case of a null rotation, (R1), we do not consider every case of axis and angle, one is sufficient;

- in case of first and second order of rotation, (R2) and (R3), we do not consider the case (a1) where $\theta$ is equal to $\frac{\pi}{2}$;

- the case (a1) where $\theta$ is equal to $\frac{\pi}{2}$ is only considered if the rotation axis and the translation direction are parallel, (Z2);

- in the case of a null translation, we do not consider any relation of orthogonality or parallelism to other directions;

- in the case of non para-perspective projection, (p1) and (p3), $\beta_{u}$ and $\beta_{v}$ are equal to zero.

We also consider the following experimental simplifications :

- when approximating a perspective projection, (p1) and (p2), we neglect the parameter $\gamma$ with respect to other approximations;

- following previous studies [8] and [26], we assume that the ratio $\frac{\alpha_{u}}{\alpha_{v}}$ is constant;

- these two previous items imply that the $\frac{\beta_{u}}{\beta_{v}}$ ratio is also constant.

Then, there only remains, from the intrinsic parameters part, 117 cases and from the extrinsic parameters part, 21709 cases, leading to a total of 2539953 particular cases. This is approximately 100 times less than previously determined (see Appendix C for details).

D R A F T April 23, 2001, 5:10pm D R A F T 


\subsection{Fundamental and homographic matrices}

For each case, we have computed the set of reduced equations. Now, for each case, we compute the fundamental or homographic matrix expression.

As previously studied in subsections $2.3,2.4$ and 2.5 , the displacements inducing homographic relations are :

- in the orthographic case (p1) : $\mathbf{u} \| O z$. The relations between $\mathbf{t}$ and $\mathbf{r}$ are equivalent to the nullity of some vector components. We will not consider (Z1) and (Z2). Previous studies on orthographic displacement have shown that the displacement is retinal $(\mathrm{t}[1 ; 3 ; 5 ; 7])$.

- in the para-perspective case (p2) : $\mathbf{u} \|\left[\begin{array}{lll}X_{0} & Y_{0} & Z_{0}\end{array}\right]$ (D2). Since the view axis has at least a component on the optical axis, we set $u_{2}= \pm 1$. Moreover the view axis is not exactly the optical axis, thus we cannot have $u_{0}=0$ and $u_{1}=0$.

- in the perspective case (p3) : $\mathbf{t}=\mathbf{0}$. Therefore we do not consider the parallelism and orthogonality constraints on $\mathbf{t}$.

We also note that, since we are dealing with only 2 views, relations between $\mathbf{r}$ or $\mathbf{t}$ with a known vector_ $\mathbf{g}$ will not simplify the H-matrix form, except in the para-perspective case, if _ $\mathbf{g}=\mathbf{M}_{\mathbf{0}}$.

The homographic relation cases lead to 351 cases of orthographic homographic relations, 18360 cases of para-perspective homographic relations and 2619 cases of perspective homographic relations, leading to a total 21330 cases of homographic relations (see explanations in Appendix C).

We will not study para-perspective and orthographic projection for fundamental matrices since the domain of validity of such projection approximations is included in conditions of existence of homographic relation. In the case of perspective projection, (p3): $\mathbf{t} \neq \mathbf{0}$ thus $u_{0}= \pm 1$ or $u_{1}= \pm 1$.

For perspective projection, there are 72252 different cases as shown in Appendix C.

\section{R A F T April 23, 2001, 5:10pm D R A F T}




\section{MATRIX FORMS}

In the previous section, we have significantly reduced the number of cases in both fundamental matrices and homographic matrices sets. However, we have still to deal with a huge amount of cases which is numerically intractable. In this section, we introduce a new idea in order to split the two sets of matrices in a two-level tree.

Each set of matrices is first split in subsets of matrices depending on their form. We determine a matrix form by a very simple parameterization. We consider $(3 \times 3)$ matrices having 9 parameters (coefficients) and we use two simple rules :

- If a coefficient is equal to zero, then there is one fewer parameter.

- If a coefficient has the same expression or is opposite to another, then there is one fewer parameter again.

These operations are very simple and can be computed on every case in a reasonable time (approximately one day for the entire process determining the matrix form).

This process, as illustrated in figure 4, reduces the 21330 cases of homography matrices to only 108 subgroups and the 72252 cases of fundamental matrices to only 188 subgroups.

The table in Appendix A shows all the particular forms of homography matrices and the table in Appendix B shows all the particular forms of fundamental matrices.

Homography and fundamental matrices are defined up to a scale factor. This parameter has not been eliminated here. We take it into account in the numerical implementation. Fundamental matrices have also to satisfy the constraint $\operatorname{det} \mathbf{F}=$ 0 . We have to check if this constraint is satisfied or not in order to know if the number of degree of freedom is reduced. This is important in order to properly use the Akaike [1] criterion at the numerical stage (see next section).

\section{R A F T April 23, 2001, 5:10pm D R A F T}




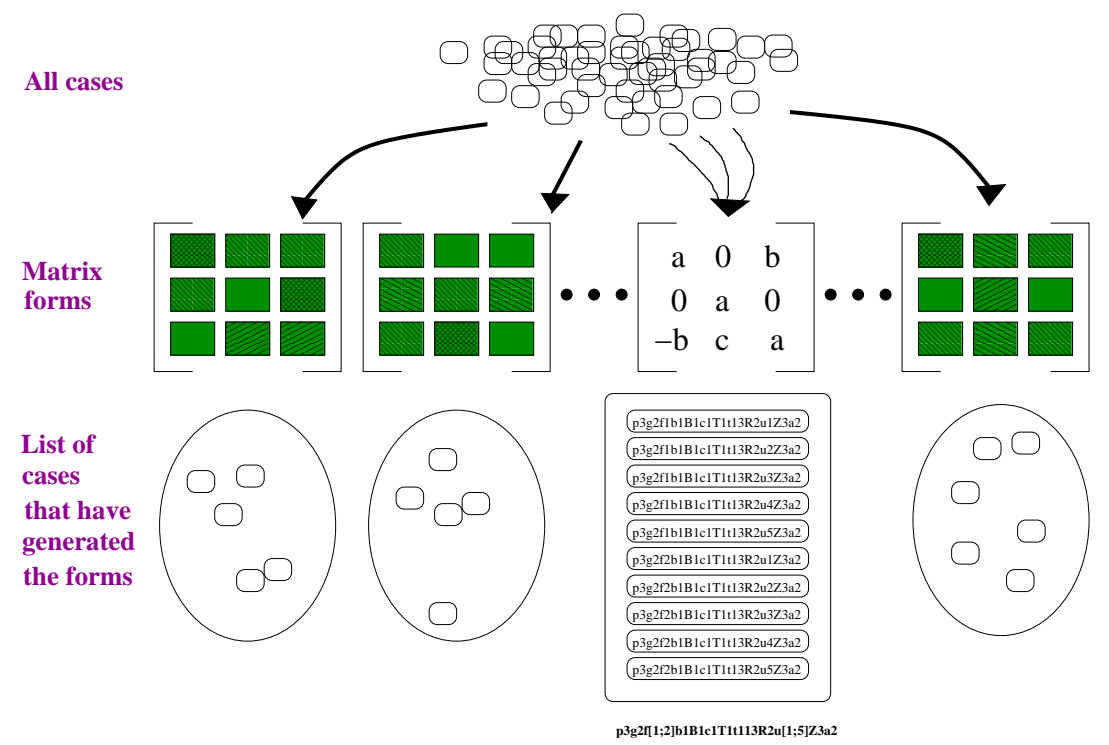

FIG. 4. Set of cases that generates the same matrix form. The central column shows an example of homography matrix form (number 19 in the table from Appendix A).

For each matrix form, we have collected all the cases that have generated them. Once the matrix form corresponding to an experiment is determined, it is possible to backtrack the source cases.

\section{EXPERIMENTS}

\subsection{Forms of homography}

We have recorded several video sequences for which the camera displacement induces a homographic relation between image points $\mathbf{m}_{\mathbf{1}}$ and $\mathbf{m}_{\mathbf{2}}$. We have first extracted points of interest and determined matching points using the image-matching algorithm from Zhang [28]. From each matrix form enumerated in table 1, we have estimated the homography parameters with the robust Least Median of Squares method [20] in order to minimize the distance between a $2 \mathrm{D}$ point $\mathbf{m}_{\mathbf{1}}$ and its
D R A F T
April 23, 2001, 5:10pm
D R A F T 
projected estimation $\mathbf{H} \mathbf{~ m}_{2}$ :

$$
\left\|\mathbf{m}_{2}-\frac{\mathbf{H} \mathbf{m}_{1}}{\left(\left(\mathbf{h}^{2}\right)^{T} \mathbf{m}_{1}\right)}\right\|^{2}
$$

where $\mathbf{h}^{2}$ represents the last line of the $\mathbf{H}$ matrix and $\mathbf{m}_{\mathbf{1}}$ and $\mathbf{m}_{\mathbf{2}}$ are normalized.

To deal with cases with different degrees of freedom, we use an appropriate Akaike criterion [1].

For each video sequence, we have verified that the model with the smallest residual error indeed corresponds to the displacement performed by a robotic system. An example is proposed in figure 5. For each pair of consecutive images, the case with the least residual error is the case $n^{\circ} 51$ in the table in Appendix A that corresponds to the matrix form :

$$
\mathbf{H}_{51}=\left(\begin{array}{ccc}
x_{1} & x_{2} & x_{3} \\
-x_{2} & x_{1} & x_{6} \\
0 & 0 & x_{1}
\end{array}\right)
$$

We observe that this case corresponds to a first order rotation (R2). If we consider only the first and the last frame, the rotation is general (R4).

We also performed several experiments without the help of a precise robotic system. A human took a camera by hand and tried to realize different particular displacements. Figure 6 shows two frames of a video sequence. The camera motion was approximately a rotation around its optical axis followed by a translation. As the previous experiment with a robotic system, for each pair of consecutive images, the case with smallest residual error is the case $n^{\circ} 51$ in the table in Appendix A. This result demonstrates the robustness of the analysis of displacement by particular cases. Even an approximative displacement is best recovered by a close particular case than the general equation.
D R A F T
April 23, 2001, 5:10pm
D R A F T 

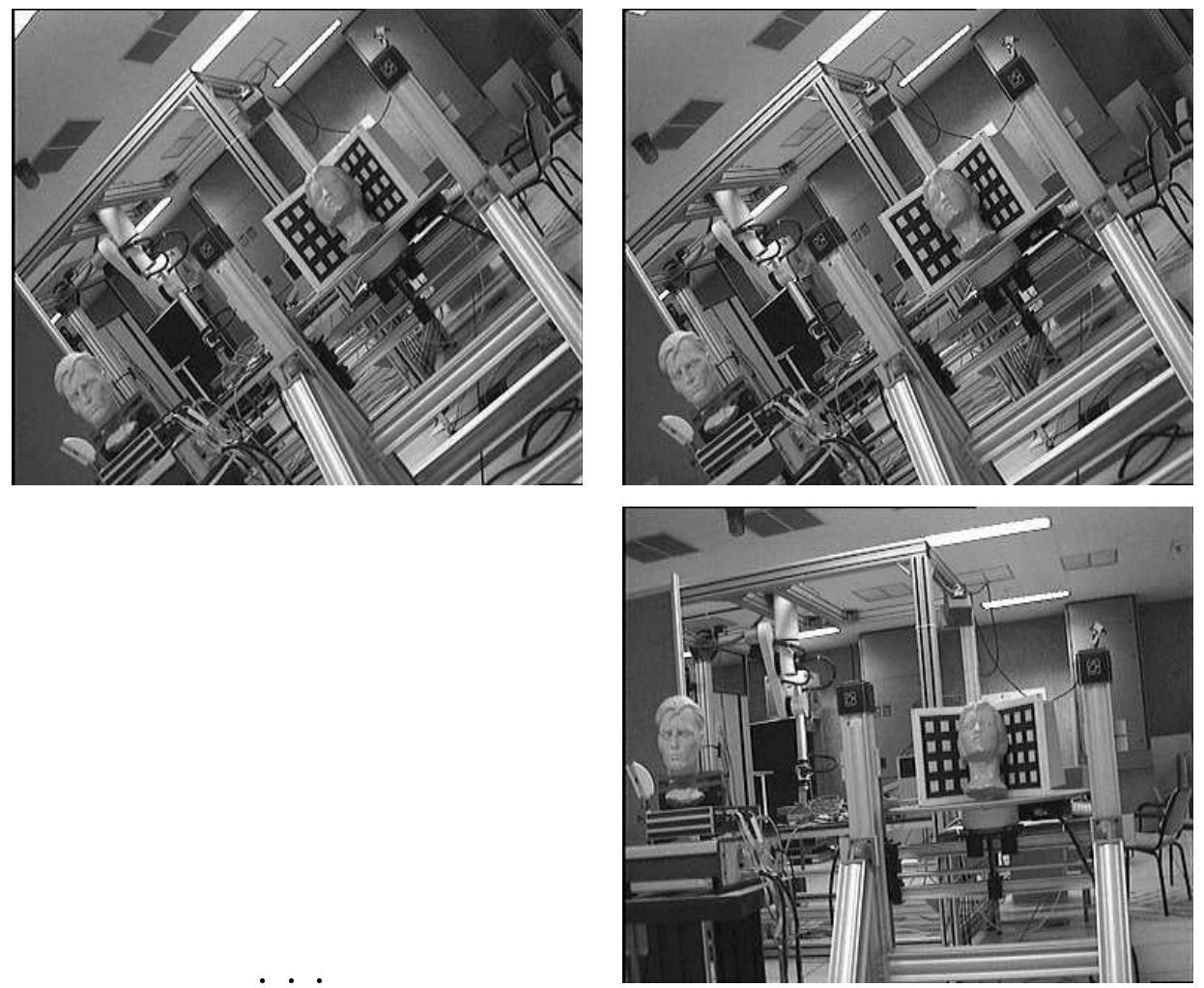

FIG. 5. Frames 1,2 and 8 of the video sequence. The robotic system performs a rotation around the optical axis.
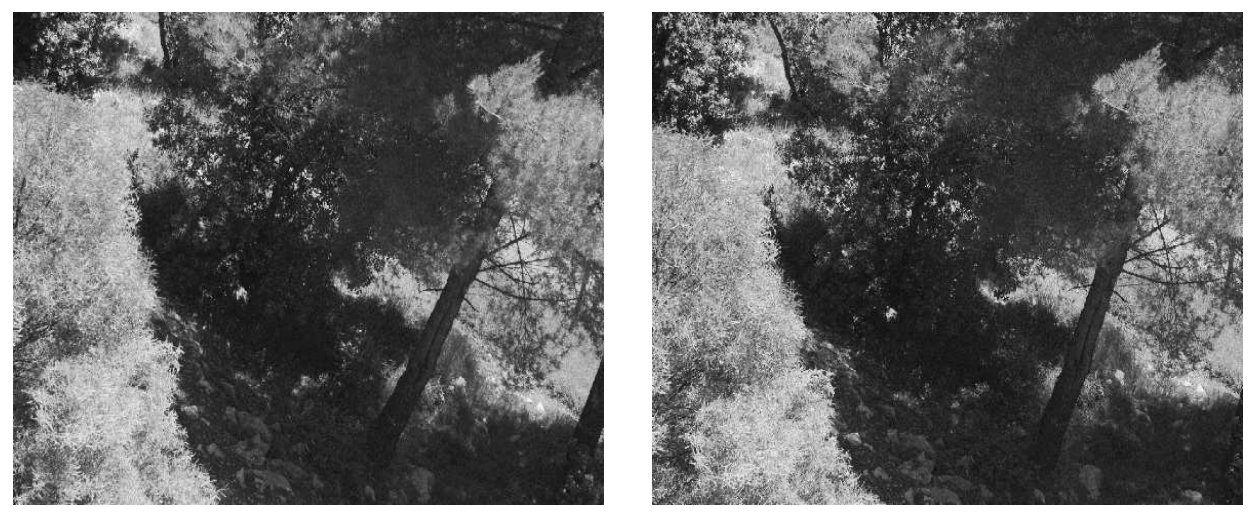

FIG. 6. Approximate rotation around the optical axis and translation.
D R A F T
April 23, 2001, 5:10pm
D R A F T 

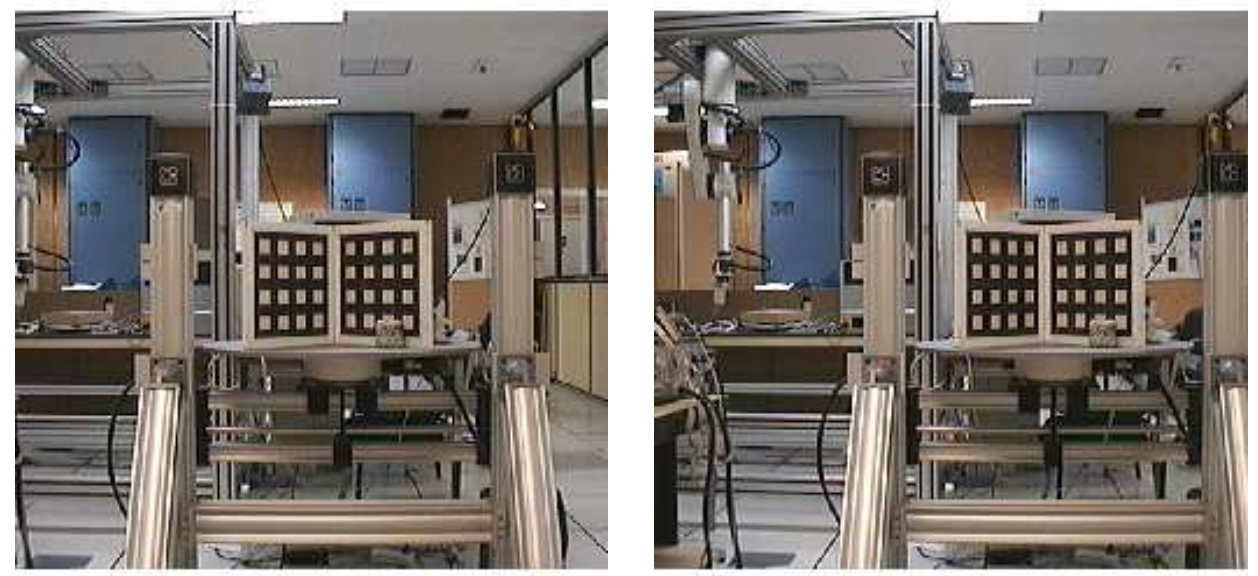

FIG. 7. x-axis translation and small pan rotation, with auto-focus

\subsection{Forms of fundamental matrices}

We have done the same experiment for a displacement that induces a fundamental relation. The criterion is using the distance between a $2 \mathrm{D}$ point $\mathbf{m}_{\mathbf{1}}$ and its epipolar line $\mathbf{F} \mathbf{m}_{\mathbf{2}}[27,16]$ :

$$
f_{m}(\mathbf{F})=\frac{\left|\mathbf{m}_{\mathbf{2}}^{T} \mathbf{F} \mathbf{m}_{\mathbf{1}}\right|}{\sqrt{\left(\mathbf{F}^{\mathbf{T}} \mathbf{m}_{\mathbf{2}}\right)_{1}^{2}+\left(\mathbf{F}^{\mathbf{T}} \mathbf{m}_{\mathbf{2}}\right)_{2}^{2}}}
$$

The camera has performed a translation parallel to the x-axis, a small pan rotation, and corrected focal length with auto-focus. The case with less residual error corresponds to the fundamental matrix form (number 59 in the table in Appendix B):

$$
\mathbf{F}_{59}=\left(\begin{array}{ccc}
0 & 0 & 0 \\
x_{0} & x_{1} & x_{2} \\
0 & -x_{2} & x_{3}
\end{array}\right)
$$

D R A F T April 23, 2001, 5:10pm D R A F T 
This particular form was obtained from cases where the rotation was approximated to its first and second order, the translation is parallel to the $\mathrm{x}$-axis, the rotation axis is orthogonal to the optical axis and the intrinsic parameters are free.

\section{HOW TO EXTEND THIS WORK TO VIDEO SEQUENCES ?}

In this paper, we have dealt with video sequences with pairs of frames. Two major extension could be down: (i) an extension of this work to trilinearities (relations between $2 \mathrm{D}$ points from 3 frames) and (ii) an extension to video sequences of $n$ frames.

In order to consider sequences of $n$ images, we need to introduce several cases about the displacements. We have constraints on translation, axis and angle of rotation and zoom factor. For each of these quantities, the questions are: is the

displacement between two frames constant? Is the acceleration constant? linear? following a known rule?

In our formalism, we need to introduce these constraints in the Maple code who will generates others equations with more constraints but also more data (n images) and more parameters. It is also easy to change the criterion used to measure the adequation of the model to the data (one $\mathrm{C}$ function to be rewritten).

We have to think about the fact that some objects may disappear along a video sequence and that their movements may be detected only in few images of the video sequences. This is a problem that will be examined in another paper.

\section{CONCLUSION}

We have studied how to deal with video sequences and with particular cases of displacement and projection that often occur in real situations (man walking on a flat road, objects far from the retina, ...). The general equations of the vision problem present singularities in some particular cases that are usually avoided.

\section{R A F T April 23, 2001, 5:10pm D R A F T}


In the present paper, we have proposed an alternative approach to this problem, using such singularities and other particular cases in order to obtain more informations than in the general case instead of avoiding them.

Our major contributions are :

- We have determined the conditions of existence of homographic relations between projected $2 \mathrm{D}$ points for the orthographic, the para-perspective and the perspective projections.

- We have used these conditions and other obvious redundancy properties to reduce the amount of homographic particular cases to study. Thus, we have determined all particular forms of matrices and we have obtained, for each particular form, the list of cases that have generated this form. This result is a first fundamental step for further studies.

This study might be extended in two ways : (i) to be able, given a form, to analyze the molecular constraints, to determine which are redundant and which correspond to the case we are dealing with, and (ii) to do the same analysis with geometrical property of the 3D scene, meaning homography induced by planes. The structure of this analysis is as general as possible to extend this work to other kind of cameras (conic mirror, ...).

The applications are twofold :

- an incremental reconstruction of the scene using different cases : each case studied has fewer parameters than the next one, giving the ability to recover some parameters from others already determined. We have already studied the control of a robot on a particular case [15].

- the segmentation of objects moving with different displacements or with different geometric properties in video sequences : using a $\nu$-trimmed square method instead of the least median square method, we can build sets of points with same matrix forms (and same numerical matrix forms).

\section{R A F T April 23, 2001, 5:10pm D R A F T}




\section{APPENDIX A}

\section{Table of particular forms of homographic matrices}

The following table shows the simplified forms obtained and, for each form, the cases that have generated them. We denote by \# the form number, by $\mathrm{p}$ the number of parameters (we have not taken into account the fact that the homography matrix is defined up to a scale factor but we do it in our numerical implementation) and by $\mathrm{n}$ the number of molecular cases that have generated a form.

\section{TABLE}

Particular forms of homography and cases that have generated them

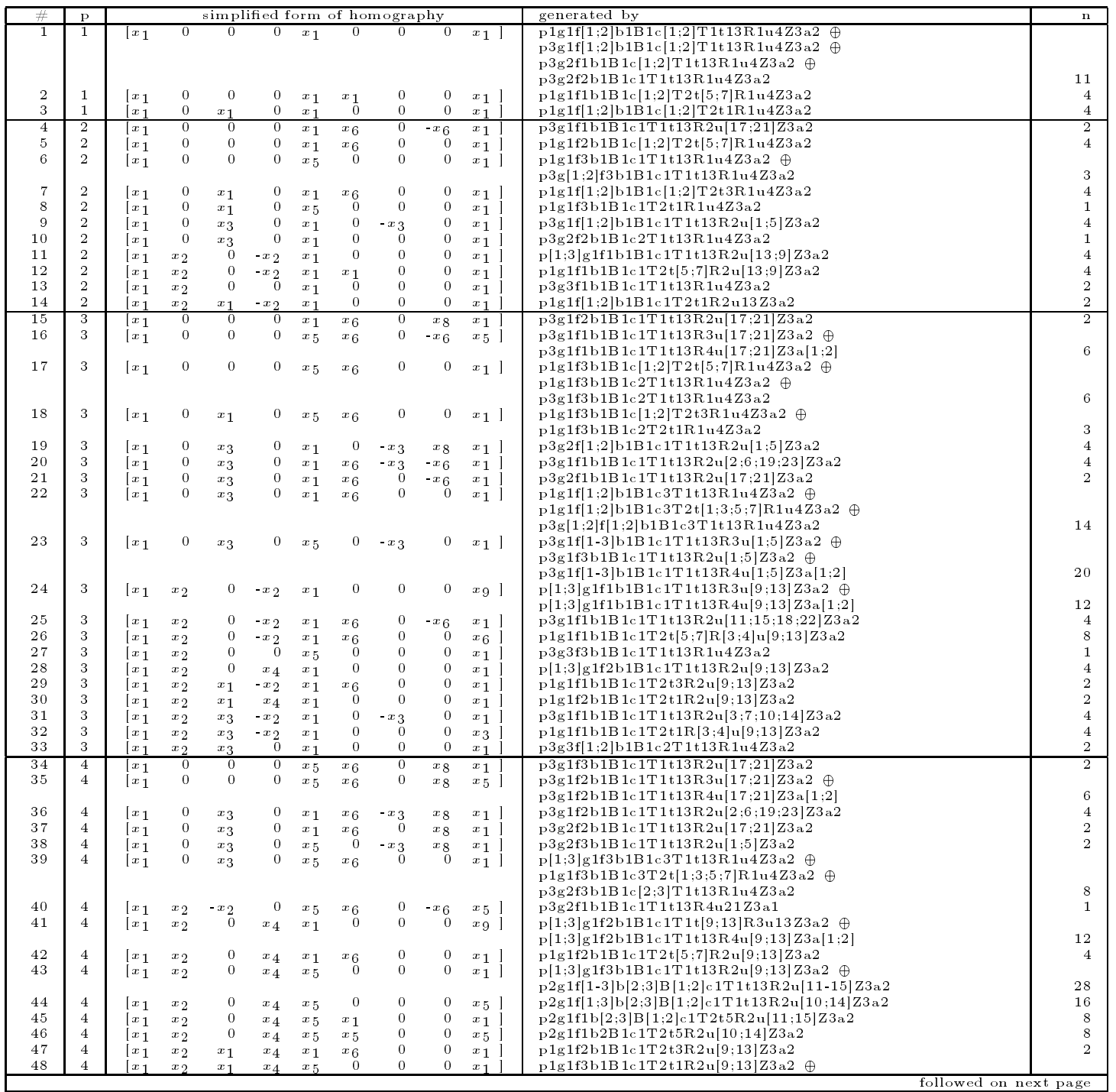
D R A F T
April 23, 2001
$5: 10 \mathrm{pm}$
D R A F T 


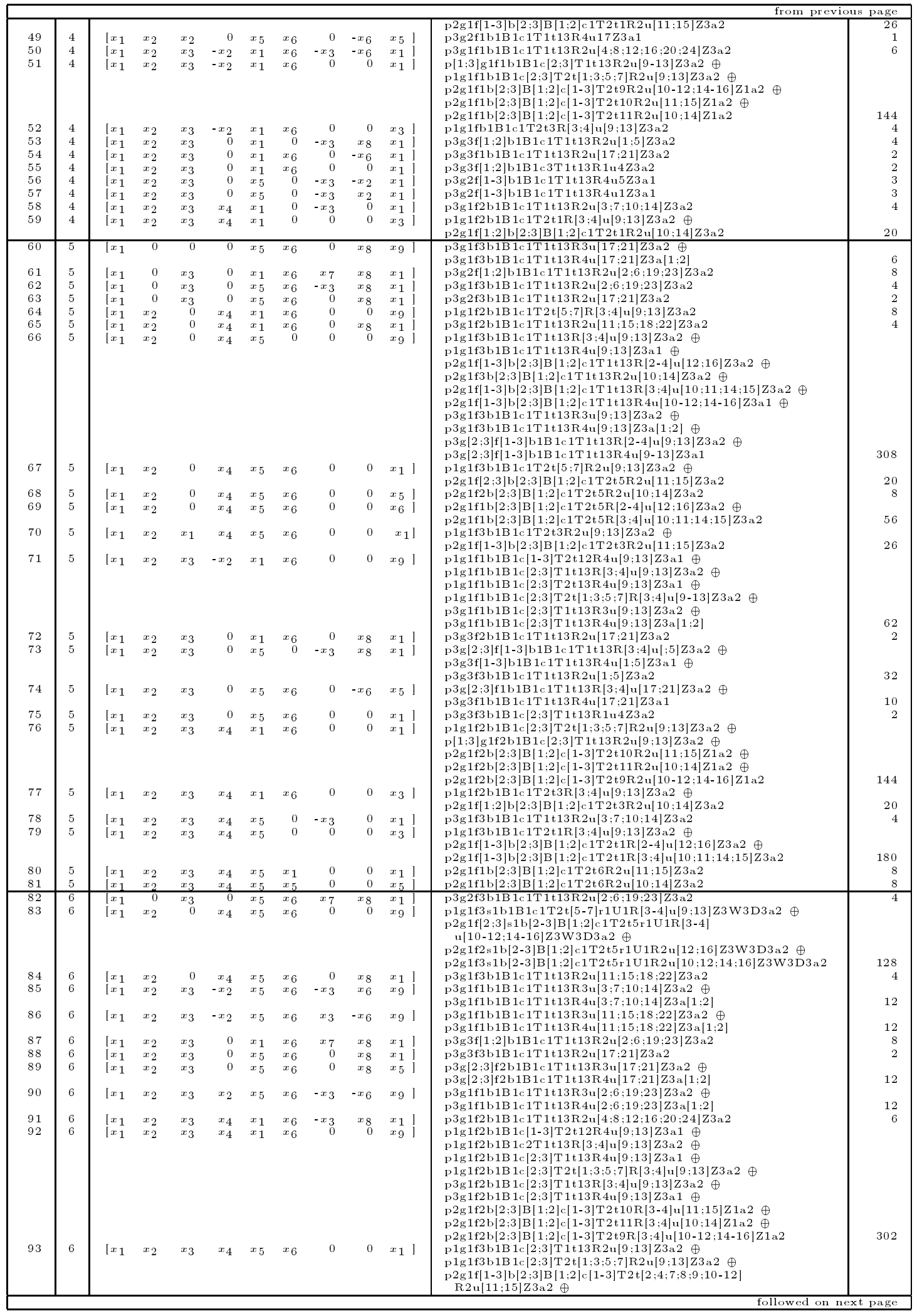

\section{R A F T April 23, 2001, 5:10pm D R A F T}




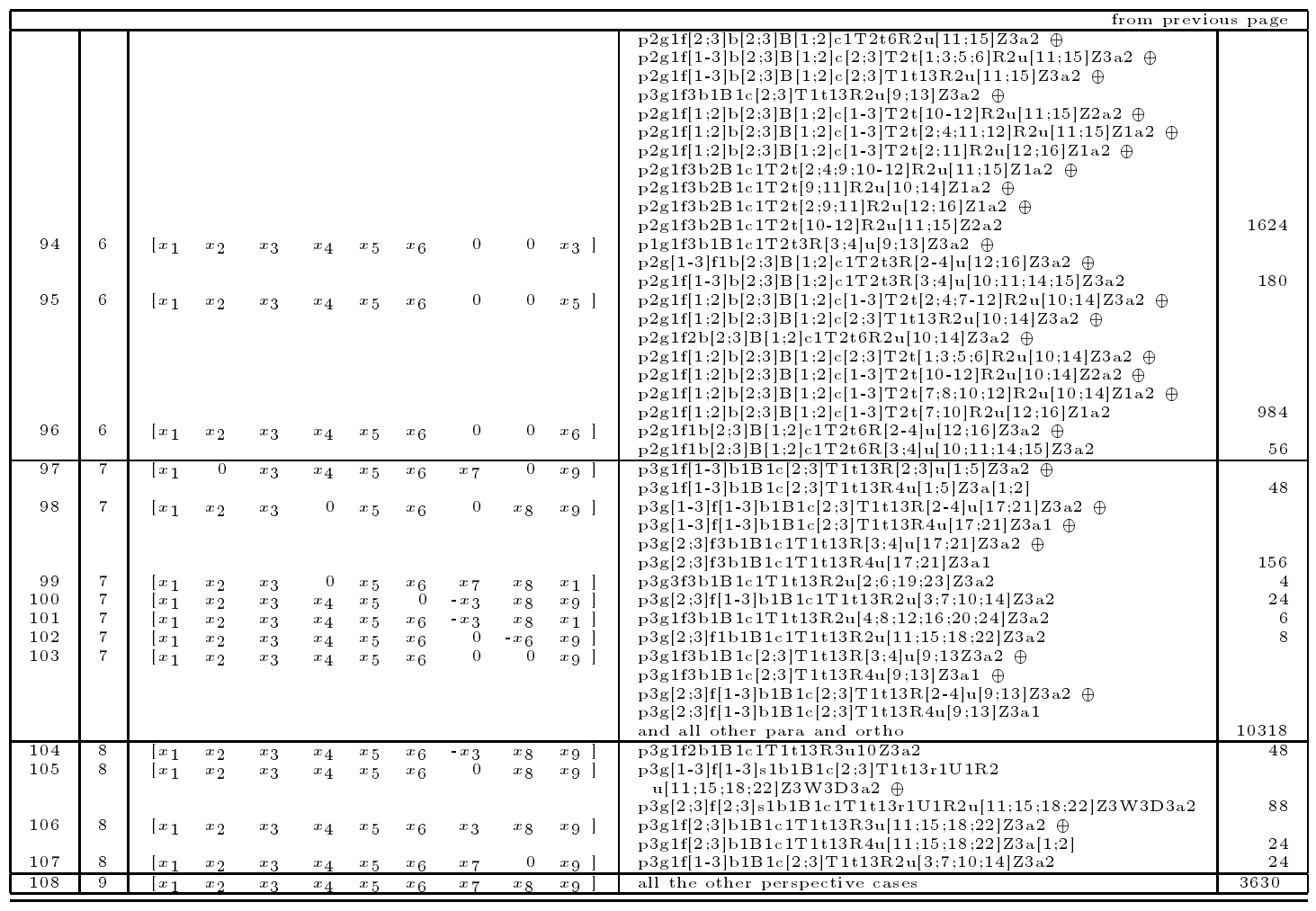

\section{APPENDIX B}

\section{Table of particular forms of fundamental matrices}

Please note that, as it is for the previous table, the number of parameters $\mathbf{p}$ does not take into account that the fundamental matrix is defined up to a scale factor and that its determinant is null. This is done is the numerical implementation.

\section{TABLE}

Particular forms of fundamental matrices

\begin{tabular}{|c|c|c|c|c|c|c|c|c|c|c|c|c|}
\hline \# & $\mathrm{p}$ & \multicolumn{9}{|c|}{ simplified form of fundamental matrices } & for example generated by: & $\mathrm{n}$ \\
\hline 1 & 1 & {$[0$} & 0 & 0 & 0 & 0 & $x_{6}$ & 0 & $-x_{6}$ & 0 & $\mathrm{~g} 1 \mathrm{f} 1 \mathrm{~s} 1 \mathrm{c} 1 \mathrm{t} 1 \mathrm{R} 1 \mathrm{u} 24 \mathrm{Z} 3 \mathrm{a} 2$ & 24 \\
\hline 2 & 1 & [0 & 0 & $x_{3}$ & 0 & 0 & 0 & $-x_{3}$ & 0 & 0 & g1f $1 \mathrm{~s} 1 \mathrm{c} 1 \mathrm{t} 5 \mathrm{R} 1 \mathrm{u} 24 \mathrm{Z} 3 \mathrm{a} 2$ & 4 \\
\hline 3 & 1 & [0 & $x_{2}$ & 0 & $-x_{2}$ & 0 & 0 & 0 & 0 & 0 & g1f1s1c1t9R1u24Z3a2 & 5 \\
\hline 4 & 2 & [0 & 0 & 0 & 0 & 0 & $x_{6}$ & 0 & $-x_{6}$ & $x_{9}$ & g1f1s1c3t1R1u24Z3a2 & 12 \\
\hline 5 & 2 & [0 & 0 & 0 & 0 & 0 & $x_{6}$ & 0 & $x_{8}$ & 0 & g1f3s1c1t1R1u24Z3a2 & 6 \\
\hline 6 & 2 & {$[0$} & 0 & 0 & 0 & 0 & $x_{6}$ & $x_{7}$ & $-x_{6}$ & 0 & $\mathrm{~g} 1 \mathrm{f} 1 \mathrm{~s} 1 \mathrm{c} 1 \mathrm{t} 1 \mathrm{R} 2 \mathrm{u} 13 \mathrm{Z} 2 \mathrm{a} 2$ & 16 \\
\hline 7 & 2 & [0 & 0 & 0 & 0 & $x_{5}$ & $x_{6}$ & 0 & $-x_{6}$ & $x_{5}$ & $\mathrm{~g} 1 \mathrm{f} 1 \mathrm{~s} 1 \mathrm{c} 1 \mathrm{t} 1 \mathrm{R} 2 \mathrm{u} 17 \mathrm{Z} 1 \mathrm{a} 2$ & 396 \\
\hline 8 & 2 & [0 & 0 & 0 & $x_{4}$ & 0 & $x_{6}$ & 0 & $-x_{6}$ & 0 & g1f1s1c1t1R2u1Z2a2 & 16 \\
\hline 9 & 2 & [0 & 0 & $x_{3}$ & 0 & 0 & 0 & $-x_{3}$ & 0 & $x_{9}$ & $\mathrm{~g} 1 \mathrm{f} 1 \mathrm{~s} 1 \mathrm{c} 3 \mathrm{t} 5 \mathrm{R} 1 \mathrm{u} 24 \mathrm{Z} 3 \mathrm{a} 2$ & 2 \\
\hline 10 & 2 & {$[0$} & 0 & $x_{3}$ & 0 & 0 & 0 & $-x_{3}$ & $x_{8}$ & 0 & $\mathrm{~g} 1 \mathrm{f} 1 \mathrm{~s} 1 \mathrm{c} 1 \mathrm{t} 5 \mathrm{R} 2 \mathrm{u} 13 \mathrm{Z} 2 \mathrm{a} 2$ & 8 \\
\hline 11 & 2 & [0 & 0 & $x_{3}$ & 0 & 0 & 0 & $x_{7}$ & 0 & 0 & g1f 1 s $2 \mathrm{c} 1 \mathrm{t} 5 \mathrm{R} 1 \mathrm{u} 24 \mathrm{Z} 3 \mathrm{a} 2$ & 4 \\
\hline 12 & 2 & {$[0$} & 0 & $x_{3}$ & 0 & 0 & $x_{6}$ & $-x_{3}$ & $-x_{6}$ & 0 & g1f1s1c1t3R1u24Z3a2 & 17 \\
\hline 13 & 2 & {$[0$} & $x_{2}$ & 0 & $-x_{2}$ & 0 & $x_{6}$ & 0 & $-x_{6}$ & 0 & g1f1s1c1t11R1u $24 Z 3 a 2$ & 8 \\
\hline 14 & 2 & [0 & $x_{2}^{2}$ & 0 & $-x_{2}$ & 0 & $x_{6}$ & 0 & 0 & 0 & $\mathrm{~g} 1 \mathrm{f} 1 \mathrm{~s} 1 \mathrm{c} 1 \mathrm{t} 9 \mathrm{R} 2 \mathrm{u} 1 \mathrm{Z} 2 \mathrm{a} 2$ & 24 \\
\hline 15 & 2 & [0 & $x_{2}$ & 0 & $-x_{2}$ & $x_{5}$ & 0 & 0 & 0 & 0 & g2f $3 \mathrm{~s} 1 \mathrm{c} 1 \mathrm{t} 9 \mathrm{R} 1 \mathrm{u} 24 \mathrm{Z} 3 \mathrm{a} 2$ & 4 \\
\hline 16 & 2 & [0 & $x_{2}$ & 0 & $x_{4}$ & 0 & 0 & 0 & 0 & 0 & g1f1s $2 \mathrm{c} 1 \mathrm{t} 9 \mathrm{R} 1 \mathrm{u} 24 \mathrm{Z} 3 \mathrm{a} 2$ & 3 \\
\hline 17 & 2 & {$[0$} & $x_{2}$ & $x_{3}$ & $-x_{2}$ & 0 & 0 & $-x_{3}$ & 0 & 0 & g1f1s1c1t10R1u24Z3a2 & 4 \\
\hline 18 & 2 & [0 & $x_{2}$ & $x_{3}$ & $-x_{2}$ & 0 & 0 & 0 & 0 & 0 & g1f1s1c1t9R2u17Z2a2 & 12 \\
\hline 19 & 2 & [0 & $x_{2}$ & $x_{3}$ & 0 & 0 & 0 & $-x_{3}$ & 0 & 0 & g1f1s1c1t5R2u17Z2a2 & 8 \\
\hline
\end{tabular}
D R A F T
April 23, 2001, 5:10pm
D R A F T 


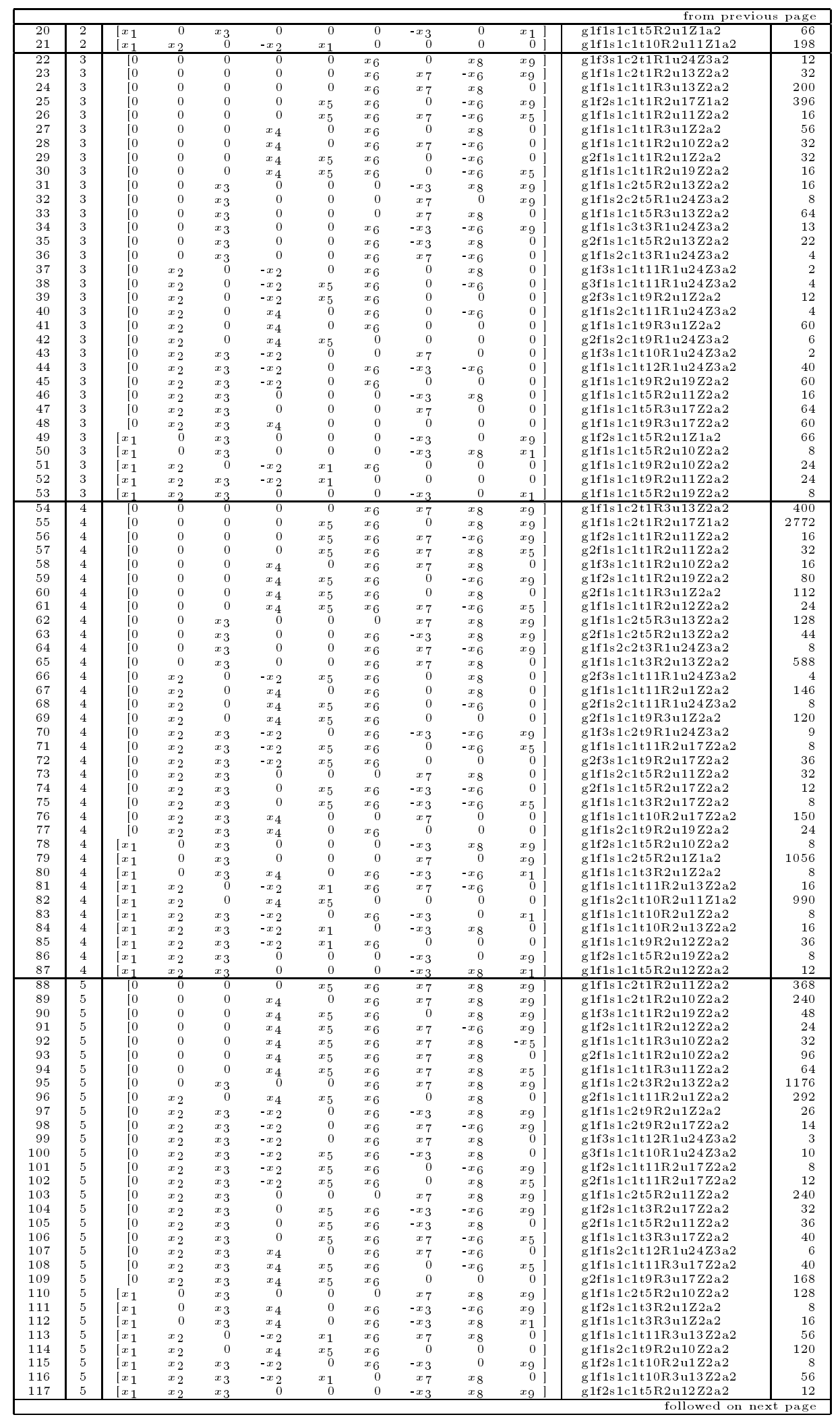
D R A F T
April 23, 2001, 5:10pm
D R A F T 


\begin{tabular}{|c|c|c|c|c|c|c|c|c|c|c|c|c|}
\hline \multicolumn{13}{|c|}{ from previous page } \\
\hline 118 & 5 & {$\left[x_{1}\right.$} & $x_{2}$ & $x_{3}$ & 0 & 0 & 0 & $x_{7}$ & 0 & $x_{9}$ & g1f1s2c1t5R2u19Z2a2 & 32 \\
\hline 119 & 5 & {$\left[x_{1}\right.$} & $x_{2}$ & $x_{3}$ & 0 & 0 & 0 & $x_{7}$ & $x_{8}$ & $-x_{1}$ & g1f1s1c1t5R3u11Z2a2 & 16 \\
\hline 120 & 5 & {$\left[x_{1}\right.$} & $x_{2}$ & $x_{3}$ & 0 & 0 & 0 & $x_{7}$ & $x_{8}$ & $x_{1}$ & g1f1s1c1t5R3u10Z2a2 & 32 \\
\hline 121 & 5 & {$\left[x_{1}\right.$} & $x_{2}$ & $x_{3}$ & $x_{2}$ & $x_{5}$ & $x_{6}$ & $-x_{3}$ & $-x_{6}$ & $x_{1}$ & $\mathrm{~g} 2 \mathrm{f} 1 \mathrm{~s} 1 \mathrm{c} 1 \mathrm{t} 5 \mathrm{R} 2 \mathrm{u} 1 \mathrm{Z} 1 \mathrm{a} 2$ & 70 \\
\hline 122 & 5 & {$\left[x_{1}\right.$} & $x_{2}$ & $x_{3}$ & $x_{4}$ & $-x_{1}$ & $x_{6}$ & 0 & 0 & 0 & g1f1s1c1t9R3u19Z2a2 & 48 \\
\hline 123 & 5 & {$\left[x_{1}\right.$} & $x_{2}$ & $x_{3}$ & $x_{4}$ & 0 & $x_{6}$ & $-x_{3}$ & 0 & $x_{1}$ & g1f1s1c1t 10R $3 u 1 Z 2 a 2$ & 16 \\
\hline 124 & 5 & {$\left[x_{1}\right.$} & $x_{2}$ & $x_{3}$ & $x_{4}$ & $x_{1}$ & $x_{6}$ & 0 & 0 & $\begin{array}{l}1 \\
0\end{array}$ & g1f1s1c1t9R $3 u 10 \mathrm{Z} 2 \mathrm{a} 2$ & 96 \\
\hline 125 & 5 & {$\left[x_{1}\right.$} & $x_{2}$ & $x_{3}$ & $x_{4}$ & $x_{5}$ & 0 & 0 & 0 & 0 & g1f1s $2 \mathrm{c} 1 \mathrm{t} 9 \mathrm{R} 2 \mathrm{u} 11 \mathrm{Z} 2 \mathrm{a} 2$ & 24 \\
\hline 126 & 6 & 10 & 0 & 0 & $x_{4}$ & $\frac{x_{5}}{x_{5}}$ & $x_{6}$ & $x_{7}$ & $x_{8}$ & $x_{9}$ & g1f1s1c1t1R3u12Z2a2 & 5160 \\
\hline 127 & 6 & [0 & $x_{2}$ & $x_{3}$ & $-x_{2}$ & 0 & $x_{6}$ & $x_{7}$ & $\begin{array}{l}8 \\
x_{8}\end{array}$ & $x_{9}$ & g1f1s1c2t9R 2 u $19 \mathrm{Z} 2 \mathrm{a} 2$ & 199 \\
\hline 128 & 6 & {$[0$} & $x_{2}$ & $x_{3}$ & $-x_{2}$ & $x_{5}$ & $x_{6}$ & $-x_{3}$ & $\begin{array}{l}x_{8} \\
\left.x^{2}-1\right)\end{array}$ & $x_{9}$ & $\mathrm{~g} 2 \mathrm{f} 3 \mathrm{~s} 1 \mathrm{c} 2 \mathrm{t} 11 \mathrm{R} 1 \mathrm{u} 24 \mathrm{Z} 3 \mathrm{a} 2$ & 34 \\
\hline 129 & 6 & [0 & $x_{2}^{2}$ & $x_{3}$ & $-x_{2}$ & $x_{5}$ & $x_{6}$ & 0 & $\begin{array}{l}x_{8} \\
\left.x^{2}-1\right)\end{array}$ & $x_{9}$ & g1f 3 s $1 \mathrm{c} 1 \mathrm{t} 11 \mathrm{R} 2 \mathrm{u} 17 \mathrm{Z} 2 \mathrm{a} 2$ & 44 \\
\hline 130 & 6 & [0 & $x_{2}^{2}$ & $x_{3}$ & $-x_{2}$ & $x_{5}$ & $x_{6}$ & $x_{7}$ & $x 8$ & 0 & g2f 3 s $1 \mathrm{c} 1 \mathrm{t} 10 \mathrm{R} 1 \mathrm{u} 24 \mathrm{Z} 3 \mathrm{a} 2$ & 10 \\
\hline 131 & 6 & [0 & $x_{2}$ & $x_{3}$ & 0 & $x_{5}$ & $x_{6}$ & $-x_{3}$ & $x_{8}$ & $x_{9}$ & $\mathrm{~g} 3 \mathrm{f} 1 \mathrm{~s} 1 \mathrm{c} 1 \mathrm{t} 3 \mathrm{R} 2 \mathrm{u} 17 \mathrm{Z} 2 \mathrm{a} 2$ & 8 \\
\hline 132 & 6 & [0 & $\begin{array}{l}x_{2} \\
x_{1}-10\end{array}$ & $x_{3}$ & 0 & $x_{5}$ & $x_{6}$ & $x_{7}$ & $-x_{6}$ & $x_{9}$ & g1f2s1c1t3R3u17Z2a2 & 40 \\
\hline 133 & 6 & [0 & $x_{2}$ & $x_{3}$ & 0 & $x_{5}$ & $x_{6}$ & $x_{7}$ & $\begin{array}{l}x_{8} \\
x^{2}-1\end{array}$ & 0 & g2f1s1c1t5R3u17Z2a2 & 192 \\
\hline 134 & 6 & {$[0$} & $x_{2}^{2}$ & $\begin{aligned} x_{3} \\
\end{aligned}$ & 0 & $x_{5}$ & $x_{6}$ & $x_{7}$ & $\begin{array}{l}\circ \\
x_{8}\end{array}$ & $x_{5}$ & $\mathrm{~g} 1 \mathrm{f} 1 \mathrm{~s} 1 \mathrm{c} 1 \mathrm{t} 3 \mathrm{R} 2 \mathrm{u} 11 \mathrm{Z} 2 \mathrm{a} 2$ & 32 \\
\hline 135 & 6 & [0 & $x_{2}$ & $x_{3}$ & $x_{4}$ & 0 & $x_{6}$ & $x_{7}$ & $\begin{array}{l}x_{8} \\
\left.x^{2}-1\right)\end{array}$ & 0 & g1f3s $2 \mathrm{c} 1 \mathrm{t} 12 \mathrm{R} 1 \mathrm{u} 24 \mathrm{Z} 3 \mathrm{a} 2$ & 3 \\
\hline 136 & 6 & {$[0$} & $x_{2}$ & $x_{3}$ & $x_{4}$ & $x_{5}$ & $x_{6}$ & 0 & 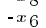 & $x_{9}$ & g1f2s1c1t11R3u17Z2a 2 & 40 \\
\hline 137 & 6 & [0 & $x_{2}$ & $x_{3}$ & $x_{4}$ & $x_{5}$ & $x_{6}$ & 0 & $\begin{array}{l}6 \\
x_{8}\end{array}$ & $x_{5}$ & g1f1s1c1t11R2u19Z2a2 & 32 \\
\hline 138 & 6 & [0 & $x_{2}$ & $x_{3}$ & $x_{4}$ & $x_{5}$ & $x_{6}$ & $x_{7}$ & $-x_{6}$ & $x_{5}$ & g1f1s1c1t12R2u17Z2a 2 & 84 \\
\hline 139 & 6 & {$\left[x_{1}\right.$} & 0 & $x_{3}$ & $x_{4}$ & 0 & $x_{6}$ & $-x_{3}$ & $\begin{array}{l}0 \\
x_{8}\end{array}$ & $x_{9}$ & g1f2s1c1t3R.3u1Z2a2 & 16 \\
\hline 140 & 6 & {$\left[x_{1}\right.$} & 0 & $x_{3}$ & $x_{4}$ & 0 & $x_{6}$ & $x_{7}$ & $\begin{array}{l}-x_{6} \\
\left.-x_{1}\right)-1\end{array}$ & $x_{9}$ & g1f 1 s $2 \mathrm{c} 1 \mathrm{t} 3 \mathrm{R} 2 \mathrm{u} 1 \mathrm{Z} 2 \mathrm{a} 2$ & 16 \\
\hline 141 & 6 & {$\left[x_{1}\right.$} & 0 & $x_{3}$ & $x_{4}$ & 0 & $x_{6}$ & $x_{7}$ & $\begin{array}{l}-x_{6} \\
-x_{6}-1\end{array}$ & $x_{9}$ & $\mathrm{~g} 1 \mathrm{f} 2 \mathrm{~s} 2 \mathrm{c} 1 \mathrm{t} 6 \mathrm{R} 2 \mathrm{u} 5 \mathrm{Z} 3 \mathrm{a} 2$ & 16 \\
\hline 142 & 6 & {$\left[x_{1}\right.$} & $x_{2}$ & 0 & $x_{4}$ & $x_{1}$ & $x_{6}$ & $x_{7}$ & $\begin{array}{l}0 \\
x_{8}\end{array}$ & 0 & g1f1s1c1t11R2u10Z2a2 & 48 \\
\hline 143 & 6 & {$\left[x_{1}\right.$} & $x_{2}$ & 0 & $x_{4}$ & $x_{5}$ & $x_{6}$ & $\begin{array}{l}7 \\
x_{7}\end{array}$ & $\begin{array}{r}-8 \\
-x_{6}\end{array}$ & 0 & g1f1s $2 \mathrm{c} 1 \mathrm{t} 11 \mathrm{R} 2 \mathrm{u} 13 \mathrm{Z} 2 \mathrm{a} 2$ & 16 \\
\hline 144 & 6 & {$\left[x_{1}\right.$} & $x_{2}$ & $x_{3}$ & $\begin{array}{l}-x_{2} \\
\left.-x_{2}\right)>1\end{array}$ & 0 & $x_{6}$ & $x_{7}$ & 0 & $x_{9}$ & g1f3s 1 c1t10R $2 u 1 Z 2 a 2$ & 8 \\
\hline 145 & 6 & {$\left[x_{1}\right.$} & $x_{2}^{2}$ & $x_{3}$ & $-x_{2}$ & $x_{1}$ & $x_{6}$ & $x_{7}$ & $x_{8}$ & 0 & $\mathrm{~g} 1 \mathrm{f} 1 \mathrm{~s} 1 \mathrm{c} 1 \mathrm{t} 12 \mathrm{R} 2 \mathrm{u} 13 \mathrm{Z} 2 \mathrm{a} 2$ & 126 \\
\hline 146 & 6 & {$\left[x_{1}\right.$} & $x_{2}$ & $x_{3}$ & $-x_{2}$ & $x_{5}$ & $x_{6}$ & $-x_{3}$ & $x_{6}$ & $x_{9}$ & g1f1s1c1t10R $2 u 10 Z 1 a 2$ & 144 \\
\hline 147 & 6 & {$\left[x_{1}\right.$} & $x_{2}$ & $x_{3}$ & $-x_{2}^{2}$ & $x_{5}$ & $x_{6}$ & $x_{3}$ & $-x_{6}$ & $x_{9}$ & $\mathrm{~g} 1 \mathrm{f} 1 \mathrm{~s} 1 \mathrm{c} 1 \mathrm{t} 11 \mathrm{R} 2 \mathrm{u} 11 \mathrm{Z} 1 \mathrm{a} 2$ & 144 \\
\hline 148 & 6 & {$\left[x_{1}\right.$} & $x_{2}$ & $x_{3}$ & 0 & 0 & 0 & $x_{7}$ & $x_{8}$ & $x_{9}$ & g1f 1 s $1 \mathrm{c} 1 \mathrm{t} 5 \mathrm{R} 3 \mathrm{u} 12 \mathrm{Z} 2 \mathrm{a} 2$ & 1536 \\
\hline 149 & 6 & {$\left[x_{1}\right.$} & $x_{2}$ & $x_{3}$ & $x_{2}$ & $x_{5}$ & $x_{6}$ & $-x_{3}$ & $-x_{6}$ & 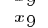 & g1f1s1c1t3R2u19Z1a2 & 358 \\
\hline 150 & 6 & {$\left[\begin{array}{ll}1 \\
x_{1}\end{array}\right.$} & $x_{2}$ & $\begin{array}{r}3 \\
x_{3}\end{array}$ & $x_{2}$ & $\begin{array}{l}5 \\
x_{5}\end{array}$ & $x_{6}$ & $\begin{array}{r}3 \\
-x_{3}\end{array}$ & $\begin{array}{r}6 \\
x_{8}\end{array}$ & $x_{1}$ & g2f1s1c1t5R2u10Z2a2 & 12 \\
\hline 151 & 6 & {$\left[x_{1}\right.$} & $x_{2}$ & $x_{3}$ & $x_{4}$ & 0 & $x_{6}$ & $-x_{3}$ & 0 & $x_{9}$ & g1f2s1c1t10R3u1Z2a2 & 16 \\
\hline 152 & 6 & {$\left[x_{1}\right.$} & $x_{2}$ & $x_{3}$ & $x_{4}$ & 0 & $x_{6}$ & $-x_{3}$ & $x_{8}$ & $x_{1}$ & $\mathrm{~g} 1 \mathrm{f} 1 \mathrm{~s} 1 \mathrm{c} 1 \mathrm{t} 12 \mathrm{R} 2 \mathrm{u} 1 \mathrm{Z} 2 \mathrm{a} 2$ & 42 \\
\hline 153 & 6 & {$\left[x_{1}\right.$} & $x_{2}$ & $x_{3}$ & $x_{4}$ & 0 & $x_{6}$ & $x_{7}$ & 0 & $x_{1}$ & g1f1s1c1t10R2u19Z2a 2 & 16 \\
\hline 154 & 6 & {$\left[\begin{array}{ll}1 \\
x_{1}\end{array}\right.$} & $\begin{array}{l}x_{2} \\
x_{2}\end{array}$ & $\begin{array}{r}3 \\
x_{3}\end{array}$ & $\begin{array}{c}x_{4} \\
x_{4}\end{array}$ & $x_{1}$ & $\begin{array}{r}{ }^{6} \\
0\end{array}$ & $\begin{array}{l}x \\
x 7\end{array}$ & $x_{8}$ & ${ }_{0}^{1}$ & g1f1s1c1t10R2u11Z2a2 & 48 \\
\hline 155 & 6 & {$\left[x_{1}\right.$} & $x_{2}$ & $x_{3}$ & $x_{4}$ & $x_{5}$ & $x_{6}$ & $-x_{3}$ & $-x_{6}$ & $x_{1}$ & g2f1s1c1t3R2u $1 \mathrm{Z} 2 \mathrm{a} 2$ & 24 \\
\hline 156 & 6 & {$\left[x_{1}\right.$} & $\begin{array}{l}x_{2} \\
x_{2}-1010\end{array}$ & $x_{3}$ & $x_{4}$ & $x_{5}$ & $x_{6}$ & 0 & $\begin{array}{r}0 \\
0 \\
0\end{array}$ & $\begin{array}{l}1 \\
0\end{array}$ & g1f1s1c1t9R3u12Z2a2 & 1428 \\
\hline 157 & 7 & 10 & $x_{2}$ & $\frac{3}{x_{3}}$ & $-x_{2}$ & $\frac{3}{x_{5}}$ & $\frac{0}{x_{6}}$ & $x_{7}$ & $x_{8}$ & $x_{9}$ & g1f1s1c2t11R2u17Z2a2 & 270 \\
\hline 158 & 7 & {$[0$} & $x_{2}$ & $x_{3}$ & 0 & $x_{5}$ & $x_{6}$ & $x_{7}$ & $\begin{array}{l}8 \\
x_{8}\end{array}$ & $x_{9}$ & g1f1s1c2t3R2u $11 \mathrm{Z} 2 \mathrm{a} 2$ & 2480 \\
\hline 159 & 7 & {$[0$} & $x_{2}$ & $x_{3}$ & $x_{4}$ & 0 & $x_{6}$ & $x_{7}$ & $\begin{array}{l}8 \\
x_{8}\end{array}$ & $x_{9}$ & g1f1s1c2t10R $2 u 17 Z 2 a 2$ & 912 \\
\hline 160 & 7 & [0 & $x_{2}$ & $x_{3}$ & $x_{4}$ & $x_{5}$ & $x_{6}$ & 0 & $\begin{array}{l}x_{8} \\
x^{2}-10\end{array}$ & $x_{9}$ & g1f2s1c1t11R2u19Z2a2 & 536 \\
\hline 161 & 7 & [0 & $x_{2}$ & $x_{3}$ & $x_{4}$ & $x_{5}$ & $x_{6}$ & $x_{7}$ & $-x_{6}$ & $x_{9}$ & g1f $2 \mathrm{~s} 1 \mathrm{c} 1 \mathrm{t} 12 \mathrm{R} 2 \mathrm{u} 17 \mathrm{Z} 2 \mathrm{a} 2$ & 84 \\
\hline 162 & 7 & {$[0$} & $\begin{array}{l}2 \\
x_{2}\end{array}$ & $x_{3}$ & $x_{4}$ & $x_{5}$ & $x_{6}$ & $x_{7}$ & $\begin{array}{l}6 \\
x_{8}\end{array}$ & 0 & g2f1s1c1t10R 2 u $17 Z 2 a 2$ & 318 \\
\hline 163 & 7 & {$\left[x_{1}\right.$} & ${ }_{0}^{2}$ & $\begin{array}{l}43 \\
x_{3}\end{array}$ & $\begin{array}{c}4 \\
x_{4}\end{array}$ & $\begin{array}{r}5 \\
0\end{array}$ & $x_{6}$ & $\begin{array}{l}7 \\
x_{7}\end{array}$ & 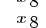 & $x_{9}$ & g1f1s1c2t3R2u10Z2a2 & 640 \\
\hline 164 & 7 & {$\left[x_{1}\right.$} & $x_{2}$ & 0 & $x_{4}$ & $x_{5}$ & $x_{6}$ & $x_{7}$ & $\begin{array}{l}x_{8} \\
\left.x^{2}-1\right)\end{array}$ & 0 & g1f1s2c1t11R2u10Z2a2 & 584 \\
\hline 165 & 7 & {$\left[x_{1}\right.$} & $x_{2}^{2}$ & $x_{3}$ & $-x_{2}$ & 0 & $x_{6}$ & $x_{7}$ & $\begin{array}{l}8 \\
x_{8}\end{array}$ & $x_{9}$ & $\mathrm{~g} 1 \mathrm{f} 1 \mathrm{~s} 1 \mathrm{c} 2 \mathrm{t} 1 \mathrm{OR} 2 \mathrm{u} 1 \mathrm{Z} 2 \mathrm{a} 2$ & 48 \\
\hline 166 & 7 & {$\left[x_{1}\right.$} & $x_{2}$ & $\begin{array}{l}x_{3} \\
x_{3}\end{array}$ & 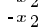 & $x_{1}$ & $x_{6}$ & $\begin{array}{l}7 \\
x_{7}\end{array}$ & $\begin{array}{l}x_{8} \\
\left.x^{2}-1\right)-1\end{array}$ & $x_{9}$ & g1f1s1c2t10R2u11Z1a 2 & 1104 \\
\hline 167 & 7 & {$\left[x_{1}\right.$} & $x_{2}$ & $x_{3}$ & 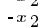 & $x_{5}$ & $x_{6}$ & $-x_{3}$ & $\begin{array}{l}8 \\
x_{8}\end{array}$ & $\begin{array}{l}9 \\
x_{9}\end{array}$ & g1f1s1c1t10R2u10Z2a2 & 32 \\
\hline 168 & 7 & {$\left[x_{1}\right.$} & $x_{2}$ & $x_{3}$ & $-x_{2}^{2}$ & $x_{5}$ & $x_{6}$ & $\vec{x} 7$ & $-x_{6}^{0}$ & $x_{9}$ & $\mathrm{~g} 1 \mathrm{f} 1 \mathrm{~s} 1 \mathrm{c} 1 \mathrm{t} 11 \mathrm{R} 2 \mathrm{u} 11 \mathrm{Z} 2 \mathrm{a} 2$ & 32 \\
\hline 169 & 7 & {$\left[x_{1}\right.$} & $x_{2}$ & $x_{3}$ & $x_{2}$ & $x_{5}$ & $x_{6}$ & $-x_{3}$ & $\begin{array}{l}0 \\
x_{8}\end{array}$ & $x_{9}$ & $\mathrm{~g} 2 \mathrm{f} 2 \mathrm{~s} 1 \mathrm{c} 1 \mathrm{t} 5 \mathrm{R} 2 \mathrm{u} 10 \mathrm{Z} 2 \mathrm{a} 2$ & 12 \\
\hline 170 & 7 & {$\left[x_{1}\right.$} & $x_{2}$ & $x_{3}$ & $x_{4}$ & 0 & $x_{6}$ & $-x_{3}$ & $\begin{array}{l}8 \\
x_{8}\end{array}$ & $x_{9}$ & g1f2s1c1t12R $2 u 1 Z 2 a 2$ & 42 \\
\hline 171 & 7 & {$\left[x_{1}\right.$} & $x_{2}$ & $x_{3}$ & $x_{4}$ & 0 & $x_{6}$ & $x_{7}$ & 0 & $x_{9}$ & g1f1s2c1t10R2u19Z2a2 & 168 \\
\hline 172 & 7 & {$\left[x_{1}\right.$} & 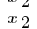 & $\begin{array}{r}3 \\
x_{3}\end{array}$ & $\begin{array}{l}x_{4} \\
x_{4}\end{array}$ & $x_{5}$ & $\begin{array}{r}6 \\
0\end{array}$ & $\begin{array}{l}7 \\
x_{7}\end{array}$ & $x_{8}$ & $\begin{array}{r}9 \\
0\end{array}$ & $\mathrm{~g} 1 \mathrm{f} 1 \mathrm{~s} 2 \mathrm{c} 1 \mathrm{t} 10 \mathrm{R} 2 \mathrm{u} 11 \mathrm{Z} 2 \mathrm{a} 2$ & 120 \\
\hline 173 & 7 & {$\left[x_{1}\right.$} & $x_{2}$ & $x_{3}$ & $x_{4}$ & $x_{5}$ & $x_{6}$ & $-x_{3}$ & $-x_{6}$ & $x_{9}$ & g1f1s1c1t3R2u19Z2a2 & 104 \\
\hline 174 & 7 & {$\left[x_{1}\right.$} & $x_{2}$ & $x_{3}$ & $x_{4}$ & $x_{5}$ & $x_{6}$ & $-x_{3}$ & $x_{8}$ & 0 & g2f1s1c1t10R2u13Z2a2 & 32 \\
\hline 175 & 7 & {$\left[x_{1}\right.$} & $\begin{array}{l}x_{2} \\
x_{2}\end{array}$ & $x_{3}$ & $x_{4}$ & $x_{5}$ & $x_{6}$ & $\begin{array}{r}-x_{3} \\
-x_{3}>101\end{array}$ & $\begin{array}{l}8 \\
x_{8}\end{array}$ & $x_{1}$ & g2f1s1c1t10R $2 u 1 Z 2 a 2$ & 262 \\
\hline 176 & 8 & $\frac{1}{10}$ & $\frac{x_{2}}{x_{2}}$ & $\frac{3}{x_{3}}$ & $\frac{x_{4}}{x_{4}}$ & $\frac{x_{5}}{x_{5}}$ & $\frac{x_{6}}{x_{6}}$ & $\frac{x_{3}}{x_{7}}$ & $\frac{x}{x_{8}}$ & $\frac{1}{x_{9}}$ & g1f1s1c2t11R2u19Z2a2 & 5220 \\
\hline 177 & 8 & {$\left[x_{1}\right]$} & $x_{2}$ & $x_{3}$ & $-x_{2}$ & $x_{5}$ & $x_{6}$ & $x_{7}$ & $x_{8}$ & $x_{0}$ & g1f1s1c2t10R2u10Z1a2 & 1232 \\
\hline 178 & 8 & {$\left[x_{1}\right.$} & $x_{2}$ & $x_{3}$ & $x_{2}$ & $x_{5}$ & $x_{6}$ & $x_{7}$ & $\begin{array}{l}8 \\
x_{8}\end{array}$ & $x_{9}$ & g1f1s1c2t3R2u $19 \mathrm{Z} 1 \mathrm{a} 2$ & 1564 \\
\hline 179 & 8 & {$\left[x_{1}\right.$} & $\begin{array}{l}2 \\
x_{2}\end{array}$ & $x_{3}$ & $x_{4}$ & $-x_{1}$ & $x_{6}$ & $x_{7}$ & 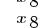 & $x_{9}$ & g1f1s1c2t9R3u19Z2a2 & 96 \\
\hline 180 & 8 & {$\left[x_{1}\right.$} & $x_{2}$ & $x_{3}$ & $x_{4}$ & 0 & $x_{6}$ & $x_{7}$ & 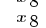 & $x_{9}$ & g1f1s1c2t10R2u19Z2a 2 & 1104 \\
\hline 181 & 8 & {$\left[x_{1}\right.$} & $x_{2}$ & $x_{3}$ & $x_{4}$ & $x_{1}$ & $x_{6}$ & $x_{7}$ & $\begin{array}{l}8 \\
x_{8}\end{array}$ & $x_{9}$ & g1f1s1c2t10R $2 u 11 \mathrm{Z} 2 \mathrm{a} 2$ & 384 \\
\hline 182 & 8 & {$\left[x_{1}\right.$} & $\begin{array}{l}x_{2} \\
x_{2}\end{array}$ & $x_{3}$ & $x_{4}$ & $x_{5}$ & $x_{6}$ & $-x_{3}$ & $\begin{array}{l}8 \\
x_{8}\end{array}$ & $x_{9}$ & g2f1s1c1t10R 2 u $10 \mathrm{Z} 1 \mathrm{a} 2$ & 774 \\
\hline 183 & 8 & {$\left[x_{1}\right.$} & $x_{2}$ & $\begin{array}{r}3 \\
x_{3}\end{array}$ & $x_{4}$ & $x_{5}$ & $x_{6}$ & $\begin{array}{r}3 \\
x_{3}\end{array}$ & $\begin{array}{l}x_{8} \\
\end{array}$ & $x_{9}$ & g2f1s1c1t11R $2 \mathrm{u} 11 \mathrm{Z} 1 \mathrm{a} 2$ & 288 \\
\hline 184 & 8 & {$\left[x_{1}\right.$} & $x_{2}$ & $x_{3}$ & $x_{4}$ & $x_{5}$ & $x_{6}$ & $x_{7}$ & $-x_{6}$ & $x_{9}$ & g1f1s2c1t11R2u11Z1a2 & 352 \\
\hline 185 & 8 & {$\left[\begin{array}{ll}1 \\
x_{1}\end{array}\right.$} & $x^{2}$ & $x_{3}$ & $x_{4}$ & $x_{5}$ & $x_{6}$ & $x 7$ & $x_{6}$ & $x_{0}$ & g1f1s $2 \mathrm{c} 1 \mathrm{t} 10 \mathrm{R} 2 \mathrm{u} 10 \mathrm{Z} 1 \mathrm{a} 2$ & 144 \\
\hline 186 & 8 & {$\left[x_{1}\right.$} & $x_{2}$ & $x_{3}$ & $\begin{array}{l}4 \\
x_{4}\end{array}$ & $x_{5}$ & $x_{6}$ & $x_{7}$ & $\begin{array}{l}6 \\
x_{8}\end{array}$ & $\begin{array}{r}9 \\
-x_{1}\end{array}$ & g2f1s1c1t5R3u11Z2a2 & 32 \\
\hline 187 & 8 & {$\left[x_{1}\right.$} & $x_{2}$ & $x_{3}$ & $x_{4}$ & $x_{5}$ & $x_{6}$ & $x_{7}$ & $\begin{array}{l}8 \\
x_{8}\end{array}$ & $\begin{array}{r}1 \\
0\end{array}$ & $\mathrm{~g} 1 \mathrm{f} 1 \mathrm{~s} 2 \mathrm{c} 1 \mathrm{t} 12 \mathrm{R} 2 \mathrm{u} 13 \mathrm{Z} 2 \mathrm{a} 2$ & 1078 \\
\hline 188 & 8 & {$\left[x_{1}\right.$} & $\begin{array}{l}x_{2} \\
x_{2}\end{array}$ & $x_{3}$ & $x_{4}$ & $x_{5}$ & $\begin{array}{l}0 \\
x_{6}\end{array}$ & $x_{7}$ & $\begin{array}{l}8 \\
x_{8}\end{array}$ & $x_{1}$ & g2f1s1c1t10R 2 u $19 \mathrm{Z} 2 \mathrm{a} 2$ & 128 \\
\hline
\end{tabular}

\section{APPENDIX C}

Details on the computations of paragraphs 3.4 and 3.5.

Here we denote by $\oplus$ the "AND" symbol and by brackets [] an interval (unix-like notation). For example, $\mathrm{p} 1 \mathrm{~g}[1-2] \oplus \mathrm{p} 2 \mathrm{~g} 3$ represents the set of the 3 cases: $\mathrm{p} 1 \mathrm{~g} 1$, p1g2 and p2g3.
D R A F T
April 23, 2001
$5: 10 \mathrm{pm}$
D R A F T 
Considering the simplification rules given in paragraph 3.4, there only remains, from the intrinsic part : p1g1f[1-3]s2b1B1c[1-3] $\oplus$ p2g1f[1-3]s2b[1-3]B[1-3]c[1-3] $\oplus$ p3g[1$3] \mathrm{f}[1-3] \mathrm{s} 2 \mathrm{~b} 1 \mathrm{~B} 1 \mathrm{c}[1-3]$

that is 117 cases. And from the extrinsic parameters part :

R1r1a2u1W3T1t1D3Z3 $\oplus$ R1r1a2u1W3T2t[1-12]D[1-3]Z3 $\oplus$ R[2-3]r1a2u[1-24]W[1-3]T1t1D3Z3 $\oplus$ R[2-3]r1a2u[1-24]W[1-3]T2t[1-12]D[1-3]Z[1-3] $\oplus$ R4r1a[1-2]u[1-24]W[1-3]T1t1D3Z3 $\oplus$ R4r1a1u[1-24]W[1-3]T2t[4;8;12]D2Z[1-3] $\oplus$ R4r1a2u[1-24]W[1-3]T2t[1-12]D[1;3]Z[1-3]

that is 21709 cases, leading to a total of 2539953 particular cases. This is approximately 100 times less than previously determined.

Continuing on paragraph 3.5 , the homographic relation cases are :

p1g1f[1-3]s1b1B1c[1-3].MVTortho

p2g1f[1-3]s1b[2-3]B[1-2]c[1-3].MVTpara

p3g[1-3]f[1-3]s1b1B1c[1-3].MVTpersp

where :

MVTpersp $=$ R1r1a2u1W3T1t1D3Z3

R[2-3]r1a2u[1-24]W3T1t1D3Z3

R4r1a[1-2]u[1-24]W3T1t1D3Z3

MVTpara $=\quad \mathrm{R}[2-3] \mathrm{r} 1 \mathrm{a} 2 \mathrm{u}[10-12 ; 14-16] \mathrm{W} 2 \mathrm{~T} 1 \mathrm{t} 1 \mathrm{D} 2 \mathrm{Z3}$

R[2-3]r1a2u[10-12;14-16]W2T2t[10-12]D2Z2

R[2-3]r1a2u[10-12;14-16]W2T2t[1-12]D2Z[1;3]

R4r1a[1-2]u[10-12;14-16]W2T1t1D2Z3

R4r1a1u[10-12;14-16]W2T2t[10-12]D2Z2

R4r1a2u[10-12;14-16]W2T2t[1-12]D2Z[1;3]

\section{R A F T April 23, 2001, 5:10pm D R A F T}


MVTortho $=$ R1r1a2u1W3T1t1D3Z3

R1r1a2u1W3T2t[1;3;5;7]D3Z3

R[2-3]r1a2u[9;13]W3T1t1D3Z3

R[2-3]r1a2u[9;13]W3T2t[1;3;5;7]D3Z3

R4r1a[1-2]u[9;13]W3T1t1D3Z3

R4r1a1u[9;13]W3T2t12D3Z3

R4r1a2u[9;13]W3T2t[1;3;5;7]D3Z3

that is 351 cases of orthographic homographic relations, 18360 cases of paraperspective homographic relations and 2619 cases of perspective homographic relations, leading to a total 21330 cases of homographic relations.but

We will not study para-perspective and orthographic projection for fundamental matrices since the domain of validity of such projection approximations is included in conditions of existence of homographic relation. In the case of perspective projection, (p3): $\mathbf{t} \neq \mathbf{0}$ thus $u_{0}= \pm 1$ or $u_{1}= \pm 1$.

As previously determined :

$$
\begin{aligned}
\text { MVTpersp }= & \text { R1r1a2u1W3T2t[1-12]D3Z3 } \\
& \text { R[2-3]r1a2u[1-24]W3T2t[1-12]D3Z[1-3] } \\
& \text { R4r1a1u[1-24]W3T2t[4;8;12]D3Z2 } \\
& \text { R4r1a2u[1-24]W3T2t[1-12]D3Z[1-3] }
\end{aligned}
$$

inducing 72252 cases of fundamental relations.

\section{REFERENCES}

1. Hirotugu Akaike. Use of an information theoretic quantity for statistical model identification. In 5th Hawaï Int. Conf. System Sciences, pages 249-250, 1972.

2. J.Y. Aloimonos. Perspective approximations. Image and Vision Computing, 8(3):179-192, August 1990 .

\section{R A F April 23, 2001, 5:10pm D R A F T}


3. Martin Armstrong, Andrew Zisserman, and Paul Beardsley. Euclidean structure from uncalibrated images. In Edwin Hancock, editor, Proceedings of the 5th British Machine Vision Conference, pages 508-518, York, UK, September 1994. BMVA Press.

4. P. Brand, R. Mohr, and P. Bobet. Distorsions optiques : correction dans un modèle projectif. Technical Report 1933, LIFIA-INRIA Rhône-Alpes, 1993.

5. L. de Agapito, E. Hayman, and I. L. Reid. Self-calibration of a rotating camera with varying intrinsic parameters. In British Machine Vision Conference, Southampton, UK, September 1998. BMVA Press.

6. D. Dementhon and L. S. Davis. Exact and approximate solutions to the three-point perspective problem. Technical Report CAR-TR-471, Computer Vision Laboratory, University of Maryland, 1989.

7. Frédéric Devernay and Olivier Faugeras. Automatic calibration and removal of distortion from scenes of structured environments. In Leonid I. Rudin and Simon K. Bramble, editors, Investigative and Trial Image Processing, volume 2567 of Proc. SPIE, San Diego, CA, July 1995. SPIE.

8. Reyes Enciso and Thierry Vieville. Experimental self-calibration from four views. In Carlo Braccini-etal, editor, 8th International Conference Image Analysis and Processing (ICIAP'95), volume 974 of Lecture Notes in Computer Science, pages 307-312, San remo, Italy, September 1995. Springer.

9. O. Faugeras. Three-Dimensional Computer Vision: a Geometric Viewpoint. MIT Press, 1993.

10. Richard Hartley. Self-calibration from multiple views with a rotating camera. In J-O. Eklundh, editor, Proceedings of the 3rd European Conference on Computer Vision, volume 800-801 of Lecture Notes in Computer Science, pages 471-478, Stockholm, Sweden, May 1994. SpringerVerlag.

11. Radu Horaud, Fadi Dornaika, Bart Lamiroy, and Stéphane Christy. Object pose: The link between weak perspective, paraperspective, and full perspective. The International Journal of Computer Vision, 22(2), 97.

\section{R A F T April 23, 2001, 5:10pm D R A F T}


12. Diane Lingrand. Analyse Adaptative du Mouvement dans des Séquences Monoculaires non Calibrées. PhD thesis, Université de Nice - Sophia Antipolis, INRIA, Sophia Antipolis, France, July 1999.

13. Diane Lingrand. Particular forms of homography matrices. In Proceedings of the 11th British Machine Vision Conference, volume 2, pages 596-605, The University of Bristol, September 2000. British Machine Vision Association, BMVA Press.

14. Diane Lingrand. Using particular forms of fundamental matrices. In James M. Ferryman and Anthony D. Worrall, editors, Proceedings of the 8th Symposium of Intelligent Robotic Systems, volume 1, pages 209-215, The University of Reading, July 2000.

15. Diane Lingrand and Thierry Viéville. Dynamic foveal 3D sensing using affine models. In Proceedings of the International Conference on Pattern Recognition, volume 1, pages 810814, Vienna, Austria, August 1996. Computer Society Press.

16. Quang-Tuan Luong, Rachid Deriche, Olivier Faugeras, and Théodore Papadopoulo. On determining the fundamental matrix: analysis of different methods and experimental results. In Israelian Conf. on Artificial Intelligence and Computer Vision, Tel-Aviv, Israel, 1993. A longer version is INRIA Tech Report RR-1894.

17. Conrad J. Poelman and Takeo Kanade. A paraperspective factorization method for shape and motion recovery. Technical Report CMU-CS-93-219, Carnegie Mellon University, School of Computer Science, December 1993.

18. Long Quan. Self-calibration of an affine camera from multiple views. The International Journal of Computer Vision, 19(1):93-105, May 1996.

19. O. Rodrigues. Des lois géométriques qui régissent les déplacements d'un système solide dans l'espace, et de la variation des coordonnées provenant de ces déplacements considérés indépendamment des causes qui peuvent les produire. Journal de Mathématiques Pures et Appliquées, 5, 1840. pp. 380-440.

20. P.J. Rousseeuw and A.M. Leroy. Robust Regression and Outlier Detection. John Wiley \& Sons, New York, 1987.

\section{R A F T April 23, 2001, 5:10pm D R A F T}


21. C. C. Slama, editor. Manual of Photogrammetry. American Society of Photogrammetry, fourth edition, 1980.

22. Stefano Soatto and Pietro Perona. Dynamic rigid motion estimation from weak perspective. In Proceedings of the 5th International Conference on Computer Vision, pages 321-328, Boston, MA, June 1995. IEEE Computer Society Press.

23. P. H. S. Torr. Geometric motion segmentation and model selection. Phil. Trans. R. Soc. Lond. A, 356:1321-1340, 1998.

24. T. Viéville. Autocalibration of visual sensor parameters on a robotic head. Image and Vision Computing, 12, 1994.

25. Thierry Viéville and Diane Lingrand. Using specific displacements to analyze motion without calibration. The International Journal of Computer Vision, 31(1):5-29, 1999.

26. Reg G. Willson. Modeling and Calibration of Automated Zoom Lenses. PhD thesis, Department of Electrical and Computer Engineering, Carnegie Mellon University, 1994. CMU-RITR-94-03.

27. Z. Zhang, R. Deriche, O. Faugeras, and Q.T. Luong. A robust technique for matching two uncalibrated images through the recovery of the unknown epipolar geometry. Artificial Intelligence Journal, 78:87-119, October 1995.

28. Zhengyou Zhang, Rachid Deriche, Olivier Faugeras, and Quang-Tuan Luong. A robust technique for matching two uncalibrated images through the recovery of the unknown epipolar geometry. Artificial Intelligence Journal, 78(1-2):87-119, 1994. Appeared in October 1995, also INRIA Research Report No.2273, May 1994.

\section{R A F T April 23, 2001, 5:10pm D R A F T}

